BNL-111805-2016-JA

\title{
Triacylglycerol Metabolism, Function and Accumulation in Plant Vegetative Tissues
}

\author{
Changcheng Xu and John Shanklin
}

Submitted to Annual Review

June 2016

Biology Department

Brookhaven National Laboratory

\section{U.S. Department of Energy DOE Office of Basic Energy Sciences}

Notice: This manuscript has been authored by employees of Brookhaven Science Associates, LLC under Contract No. DE- SC00112704 with the U.S. Department of Energy. The publisher by accepting the manuscript for publication acknowledges that the United States Government retains a non-exclusive, paid-up, irrevocable, world-wide license to publish or reproduce the published form of this manuscript, or allow others to do so, for United States Government purposes. 


\section{DISCLAIMER}

This report was prepared as an account of work sponsored by an agency of the United States Government. Neither the United States Government nor any agency thereof, nor any of their employees, nor any of their contractors, subcontractors, or their employees, makes any warranty, express or implied, or assumes any legal liability or responsibility for the accuracy, completeness, or any third party's use or the results of such use of any information, apparatus, product, or process disclosed, or represents that its use would not infringe privately owned rights. Reference herein to any specific commercial product, process, or service by trade name, trademark, manufacturer, or otherwise, does not necessarily constitute or imply its endorsement, recommendation, or favoring by the United States Government or any agency thereof or its contractors or subcontractors. The views and opinions of authors expressed herein do not necessarily state or reflect those of the United States Government or any agency thereof. 


\title{
Triacylglycerol Metabolism, Function and Accumulation in Plant Vegetative Tissues
}

\author{
Changcheng Xu and John Shanklin
}

Biological, Environmental and Climate Sciences Department, Brookhaven National Laboratory, Upton, NY 11973; email: cxu@bnl.gov, shanklin@bnl.gov

Keywords: Triacylglycerol, fatty acid, $\beta$-oxidation, lipid homeostasis, metabolic engineering

\begin{abstract}
Oils in the form of triacylglycerols are the most abundant energy-dense storage compounds in eukaryotes and their metabolism plays a key role in cellular energy balance, lipid homeostasis, growth and maintenance. Plants accumulate oils primarily in seeds and fruits. Plant oils are used for food and feed, and increasingly as feedstocks for biodiesel and industrial chemicals. While plant vegetative tissues do not accumulate significant levels of triacylglycerols, they possess a high capacity for their synthesis, storage and metabolism. The development of plant vegetative oil accumulation presents an opportunity to create novel renewable platforms for expanded production of triacylglycerols as a renewable and sustainable bioenergy source. Here, we review recent progress in the understanding of triacylglycerol synthesis, turnover, storage and function in leaves, and discuss emerging genetic engineering strategies targeted at enhancing triacylglycerol accumulation in biomass crops. Such a platform could potentially be modified to produce desired oleochemical feedstocks or nutraceuticals.
\end{abstract}

Abbreviations: FA, fatty acid; TAG, triacylglycerol; DW, dry weight; WRI, WIRNKLED; ACCase, acetyl-CoA carboxylase; BCCP, biotin carboxyl carrier protein; BC, biotin carboxylase; MCT, medium chain thioesterase; FAT, fatty acid thioesterase; TGD1, trigalactosyldiacylglycerol1; DAG, diacylglycerol; PA, phosphatidic acid; PC, phosphatidylcholine; DGAT, diacylglycerol:acyl-CoA acyltransferase; PDAT, phospholipid:diacylglycerol acyltransferase; LD, lipid droplet; SDP, sugar-dependent; PXA, peroxisomal ATP binding cassette transporter; LPCAT, acyl-CoA:lysophosphatidylcholine acyltransferase; LEC, leafy cotyledon. 


\section{Introduction}

Fatty acid (FA) synthesis occurs in every cell of the plant. However, while vegetative organs such as stems and leaves accumulate substantial levels of membrane and surface lipids, triacylglycerols (TAG) are barely detectable; typically accumulating to $<0.1 \%$ of dry weight (DW). This is because leaves evolved as source tissues highly specialized for photosynthetic carbohydrate synthesis and export for metabolism, growth, and energy storage (30). As illustrated in Figure 1, during photosynthesis, light energy is converted to chemical energy, which is used to fuel the synthesis of carbohydrates from carbon dioxide by the Calvin cycle in the stroma of chloroplasts. This results in the production of triose phosphates (TPs). These TPs can be used to synthesize starch, lipids and amino acids in the chloroplast or be exported to the cytosol by the triose phosphate transporter (TPT) for sucrose synthesis. The partitioning of newly assimilated carbon between various metabolic pools is a dynamic processes that is controlled in part by metabolic status, circadian clock and redox cascade $(53 ; 91 ; 194)$. While sucrose represents the major long-distance transport form of photoassimilates, up to half of the carbon fixed by photosynthesis is transiently stored as starch in the chloroplast in mature leaves. This starch is hydrolyzed to sugars primarily destined for export at night $(52 ; 59 ; 165)$; indeed, up to $80 \%$ of the carbon assimilated during photosynthesis is exported from mature leaves via phloem to satisfy the metabolic and energy storage needs of sinks (76). In seeds and fruits, sucrose is cleaved into hexoses for the synthesis of starch, storage proteins and oils. Of these, oils in the form of TAGs contain more than twice the energy density of equivalent masses of carbohydrates and proteins. TAGs serve multiple important roles in plants. They are the major reserves of FAs for energy production and the synthesis of carbohydrates during seed germination and early seedling establishment $(56 ; 169)$ and are essential for normal growth and development of adult plants (196). In addition, recent studies show that TAGs function as a buffer for cytotoxic FAs and other lipid intermediates, thereby playing a key role in intracellular lipid homeostasis and cell survival $(43 ; 44)$.

Plant oils are important sources of food, feed and industrial feestocks for a variety of applications including the production of biodiesel, a mixture of FA esters derived from TAGs. Currently, biodiesel is produced from conventional oil crops. Oilseed-based fuel production, however, can only meet a small proportion of world transportation needs before impinging on the global food supply (38). One solution for expanding plant oil production in an environmentally sustainable way is to exploit the production of oil in vegetative biomass $(30 ; 175)$. This is because: 1) Vegetative tissues of sustainable energy crops such as perennial grasses and poplar are far more abundant than seeds and fruits; therefore, producing oil in vegetative tissues, especially has the potential to yield large quantities of oil from a modest land area. For example, the high biomass yields of sugarcane of approximately 20 ton/acre, even with a modest TAG yield of $1.5 \%$ of dry

mass would yield approximately 100 gal of oil/acre, which is equivalent of the average yield of an acre of canola grown in North Dakota. Based on published studies from model plants (43; 180 ), a yield of $15 \%$ oil by per dry weight may be achievable in crop plants using a combination 
of genetic strategies. One acre of such a crop could therefore displace approximately 10 acres of canola. 2) Increasing oil content enhances the energy density and nutritional value of biomass, which is beneficial for producing electricity through burning, renewable fuels through thermochemical conversion, or nutritionally enhanced animal feed $(26 ; 30 ; 38 ; 125 ; 127) .3)$ Producing oil in vegetative tissues may not compete with food production. Together these drivers are fueling interest in understanding the pathways and regulation of plant vegetative TAG metabolism, and the exploitation of this knowledge to engineer a new generation of oilaccumulating biomass crops.

Several reviews on metabolic engineering strategies to achieve vegetative-oil accumulation have recently been published $(26 ; 30 ; 121 ; 175 ; 182)$. Here, we summarize current understanding of TAG synthesis, turnover, storage and function in leaves, and discuss recent advances in metabolic engineering efforts and perspectives for the development of novel bioenergy crops. Where appropriate, information from studies of sink organs i.e., seeds is compared and contrasted with studies of source tissues exemplified by leaves.

\section{Triacylglycerol Biosynthesis}

\subsection{Carbon flux to FAs}

Plant vegetative tissues contain 5-10\% FAs on a dry weight basis; the majority of which are found in membrane lipids (126). Indeed, in a number of different studies the FA content in TAG represent less than $0.05 \%$ per dry weight in wild-type Arabidopsis leaves $(43 ; 145 ; 162 ; 193)$. In higher plants, FAs are synthesized almost exclusively in the plastid and pyruvate is the primary precursor for FA synthesis. In oilseeds, hexoses generated by sucrose cleavage are metabolized into pyruvate for FA synthesis via the glycolysis pathway, which operates in parallel in both the cytosol and the plastid $(8 ; 15 ; 16)$. This parallel pathway can interact through the action of highly selective transporters present in the inner envelope of plastids including the phosphoenolpyruvate (PEP)/phosphate transporter (PPT) and hexose phosphate transporter (187) (Figure 2). In Brassicaceae, such as canola (Brassica napus) or Arabidopsis, it is generally believed that the cytosolic glycolytic pathway is the major route for the metabolism of hexoses up to PEP, whereas the plastidial counterpart is largely responsible for conversion of PEP to pyruvate catalyzed by pyruvate kinase. However, disruption of PPT in the plastid envelope results in no significant changes in FA content in Arabidopsis seeds (99; 134), suggesting alternative routes for carbon import into plastids from the cytosol for FA synthesis at the level of hexose phosphates $(77 ; 122)$. In contrast to sink tissues such as seeds and roots, the hexose phosphate transport activities are not detectable in leaf mesophyll cells (122). In addition, the glycolytic pathway in leaf chloroplasts seems to be incomplete, since the single-copy gene ENO1, which encodes the plastid-localized enolase responsible for the conversion of 2phosphoglycerate into PEP via glycolysis, is not expressed in leaves $(46 ; 134 ; 135)$. A plastid- 
localized sodium-dependent pyruvate transporter (BASS2) has recently been identified and disruption of this transporter in Arabidopsis appears to only affect plastid-localized isopentenyl diphosphate synthesis, but not the vegetative or reproductive growth of plants (51), therefore pyruvate generated via cytosolic glycolysis is rather unlikely to be the major source of carbon for FA synthesis in plant vegetative tissues. Together, these results suggest that PEP derived from cytosolic glycolysis represents the main carbon source for FA synthesis in chloroplasts of leaf mesophyll cells.

\subsection{Role of WRI transcription factors in the regulation of carbon flux to FAs}

Carbon metabolism in seeds is tightly regulated at the level of transcription by an array of transcription factors that coordinate storage compound accumulation, embryogenesis and seed maturation. Among them, WIRNKLED1 (WRI1) is a member of the APETALA2-ethyleneresponsive element binding protein (AP2/EREBP) subfamily involved in the transcriptional regulation of carbon partitioning into FA synthesis in seeds. Mutations in WRI1 cause a severe reduction in carbon flux from sugars to pyruvate in the plastidial glycolytic pathway $(47 ; 109)$ and a $80 \%$ decrease in FA content in seed TAG $(17 ; 28 ; 47)$. It appears that inactivation of WRI1 causes severe reductions in the activity of several glycolytic enzymes, but labeling experiments implied that FA synthesis is not affected in the mutant seeds (47). Interestingly, transcriptomic analysis showed that genetic modifications of WRI1 not only affect the transcript levels of genes involved in late steps of glycolysis but also those in the plastidial FA synthesis pathway $(17 ; 113 ; 143 ; 176)$. Transient overexpression of WRI1 in N. Benthamiana leaves resulted in the upregulation of transcripts encoding enzymes involved in plastid uptake and metabolism of phosphoenolpyruvate, fatty acid and oil biosynthesis and fatty acid degradation, whereas those related to photosynthesis and starch degradation were down-regulated (60).

Most studies on the regulation of FA synthesis focus on acetyl-CoA carboxylase (ACCase), which catalyzes a potential rate-limiting step in FA synthesis in plastids (128). Plastidial ACCase is a heteromeric protein complex consisting of four different proteins: an $\alpha$-subunit of carboxyltransferase ( $\alpha-\mathrm{CT})$, a biotin carboxyl carrier protein (BCCP), a biotin carboxylase (BC) and a $ß$-subunit of carboxyltransferase (ß-CT). During the stages of rapid seed filling and vegetative plant growth, all these four ACCase subunits are coordinately expressed to meet the increased cellular demand for FAs $(80 ; 143)$ and overexpression or downregulation of single subunits often leads to deleterious consequences on plant growth and development, but has only minor effects on FA accumulation in seeds $(32 ; 168)$. WRI1 overexpression or disruption appears to not affect all the subunits of the plastidial ACCase at transcript $(17 ; 143)$ and protein (49) levels, raising an intriguing question as to whether changes in ACCase activity per se can explain the oil phenotypes associated with wri1 mutants or in plants overexpressing WRI1. 
Besides WRI1, the Arabidopsis genome encodes three other WRI-like genes including WRI3 and WRI4 (174). Overexpression of WRI3 and WRI4 rescues the low oil accumulation phenotype of wri1 mutants, demonstrating the in vivo functionality of these two WRI-like genes in regulating carbon partitioning into FA synthesis. While WRI1 is specifically expressed in seeds, WRI3 and WRI4 appear to be more ubiquitously expressed, with the highest transcripts found in vegetative tissues and flowers. Disruption of WRI3 and/or WRI4 does not affect seed oil accumulation, but all three WRIs are required in floral tissues to provide precursors for cutin biosynthesis and to prevent the fusion of flower organs during flower development. However, triple mutants lacking all three WRIs display no obvious alteration in vegetative growth, suggesting the involvement of other unknown transcription or other factors in regulating carbon partitioning to FA synthesis in vegetative tissues $(174 ; 176)$.

\subsection{Biochemical regulation of FA synthesis}

Recent work uncovered a complex network of biochemical regulation that provides a number of potential targets for biotechnological manipulation, see Figure 3. For example, ACCase activity is strongly induced in the light, a phenomenon that has been attributed to changes in plastidial redox status mediated by thioredoxins (149). Downregulation of ACCase has also been demonstrated by a proteinaceous regulator PII, the sequence of which is conserved between archaebacteria, bacteria, and plants, although interestingly, the physiological functions of their homologs are not conserved. In plants the ATP-activated PII binds to the BCCP subunit of ACCase causing its inactivation (22). To underscore the complexity of ACCase's regulatory network, synthesis of the inhibitory PII protein is controlled by the oil-promoting transcription factor WRI1, which also induces its target BCCP. This apparent paradox has been interpreted as a PII-mediated fine-tuning mechanism (14). A regulatory circuit for ACCase was proposed by Ohlrogge based on Tween-feeding experiments (158). Subsequent work demonstrated the plastidial ACCase is specifically inhibited by oleoyl-acyl carrier protein (ACP), the terminal product of the ACP track of plastidial FA biosynthesis by a rapid and reversible end-product inhibition (2). In the same work the authors described a later, irreversible phase of ACCase inhibition, implying several distinct mechanisms occur on different timescales. The long term inhibition could be mediated by posttranslational modifications; and while such inhibitory modifications have yet to be identified in plant ACCase, two regulatory phosphorylation sites were identified in the yeast ACCase which, when mutated resulted in increased ACCase activity and total FA content (154).

Expression of a medium chain-acyl carrier protein thioesterase (MCT) in Brassica napus resulted in the accumulation of lauric acids in TAG along with increases in both FA synthesis and $\beta$ oxidation (42). The levels of several FA biosynthetic enzymes increased and the levels of long 
chain acyl-ACPs declined. It is likely that a combination of decreased oleoyl-ACP and increases in the amount of several biosynthetic enzymes contributed to the increase in FA synthesis. Interestingly, coexpression of the four ACCase subunits in cyanobacteria in combination with overexpression of an MCT resulted in efficient chain termination of medium chain FAs, and their secretion into the media was stimulated by separate changes that weakened the cell wall (106). This strategy essentially phenocopied that of the Brassica MCT overexpression in which FA synthesis was upregulated upon chain termination/export from the plastid (42). In theory, overexpressing FATA or FATB, i.e., the oleoyl-ACP or palmitoyl-/oleoyl-ACP thioesterases (183), respectively, could stimulate ACCase via reducing oleoyl-ACP inhibition, though combining such overexpression with disruptions of acyl-ACP synthases (AAE15/16) (92) has been shown to partially prevent the re-esterification of FAs to ACP (172). Disrupting major isoforms of the stearoyl-ACP desaturase (SAD) such as SSI2 (75) represents another potential approach to reducing oleoyl-ACP levels while increasing those of stearoyl-ACP. However, as of yet, none of these approaches has been reported to increase oil accumulation in seeds.

Mutations in trigalactosyldiacylglycerol1 (TGD1), a component of the $\mathrm{ABC}$ transporter responsible for the reimport of phospholipids into the plastid results in decreased lipid availability for photosynthetic membrane synthesis (191). It was recently shown that relative to

WT, tgd1 exhibits a 3.8-fold increase in FA biosynthesis (44). Alternatively, stimulating FA export by overexpression of a recently-identified FA export protein 1 (FAX1) resulted in a decrease in the levels of plastid lipids and increased TAG accumulation in leaves and flowers (100). Together these data show that decreasing plastid FA levels either by increasing their export or decreasing their reimport stimulates FA synthesis.

\subsection{Regulation of FA partitioning between TAG and membrane lipids}

In photosynthetic tissues of most plants, the end products of FA synthesis have two metabolic fates. They can be used directly in the plastid for the sequential acylation of glycerol-3phosphate (G3P) catalyzed by plastidial acyltransferases, leading to the generation of phosphatidic acid (PA). Dephosphorylation of PA by PA phosphohydrolase (PAH) gives rise to diacylglycerol (DAG). Alternatively, FAs are exported from the plastid and first incorporated into phosphatidylcholine (PC) through a very active deacylation/ reacylation cycle (12), with acyl-CoA:lysophosphatidylcholine acyltransferases (LPCATs) playing a central role (94). Acyl groups released from $\mathrm{PC}$ acyl editing rather than nascent FAs directly exported from the plastid are used for the synthesis of PA and DAG by G-3-P acylation and PA dephosphorylation reactions, respectively in the ER. DAG is the common precursor of TAG and membrane lipids. However, in leaves, the majority of DAG assembled from de-novo synthesized FAs is used for membrane lipid synthesis to support cell growth, proliferation and membrane maintenance (9). 
In addition to serving as central intermediates in lipid metabolism, both PA and DAG play important roles in lipid signaling. While the signaling function of DAG appears to be largely limited to higher eukaryotes (27), PA is a universal signaling molecule implicated in a wide variety of cellular processes including lipid metabolism, vesicular trafficking and membrane biogenesis (108). In eukaryotes, key players involved in the control of PA and DAG levels are the lipin family of $\mathrm{Mg}^{2+}$-dependent PAHs. In yeast, DAG pools generated by the lipin orthologue pah1p, are the major source of DAG for TAG synthesis and inactivation of pahlp causes a dramatic reduction in TAG synthesis and storage $(1 ; 65)$ and an increase in accumulation of fatty acids, phospholipids and sterol esters $(64 ; 65 ; 147)$. Similarly, disruption of the single lipin orthologue in Drosophila melanogaster (177) or inactivation of lipin-1 in mice (116) or lipin-2 in human cells (178) leads to defects in TAG storage. Arabidopsis contains two lipin homolog proteins called PAH1 and PAH2 (119). Disruption of both genes leads to increased FA and phospholipid synthesis, massive proliferation of endoplasmic reticulum (ER) membranes (33; 41) and decreased TAG accumulation in leaves (43), suggesting a conserved role for lipins in triacylglycerol synthesis for yeast, mammals and plant vegetative tissues.

Membranes of the lipin family are bifunctional intracellular proteins, acting as transcriptional regulators in controlling the expression of genes involved in membrane and storage lipid metabolism, in addition to catalyzing the dephosphorylation of PA $(66 ; 69 ; 132)$. However, the up-regulation of phospholipid synthesis gene expression in yeast mutant cells lacking pahlp is due the accumulation of the pahlp substrate PA (64). The transcriptional regulatory functions of mammalian lipins are also mediated by PA $(39 ; 116 ; 197)$. Similarly, increased phospholipid synthesis in Arabidopsis mutants disrupted in PAH1 and PAH2 is due to the biochemical activation of the key enzyme in the PC biosynthetic pathway by PA accumulation rather than to the increased expression of genes associated with phospholipid synthesis (33). Thus, a picture is rapidly emerging that PA plays a central signaling role in regulating lipid metabolism and that lipid family proteins are key determinants of levels of a PA pool that impacts FA partitioning between membrane lipid and TAG synthesis.

\subsection{Relative contributions of acyl-CoA-dependent and -independent pathways to TAG synthesis}

The esterification of DAG to TAG represents the last and the only committed step in de novo TAG synthesis. In plants, although plastids also contain TAG and harbor TAG synthetic activities, ER is the major site of TAG assembly and DAG originating from ER is the predominant precursor for TAG synthesis. In support of the ER-localization of TAG biosynthesis, key enzymes involved in DAG synthesis and the final step of TAG synthesis are also associated with the ER network $(13 ; 31)$. In contrast to most seeds, where PC is the major donor of DAG for TAG synthesis (13), PA dephosphorylation catalyzed by the Arabidopsis lipins appears to the major source of DAG for TAG synthesis in leaves (43) (Figure 4). In yeast, 
plants and algae, DAG esterification to TAG can proceed via both acyl-CoA-dependent and independent mechanisms $(13 ; 31)$. The acyl-CoA-dependent TAG synthesis is catalyzed by diacylglycerol:acyl-CoA acyltransferases (DGATs) using acyl-CoA and DAG as substrates to form TAG. In plants, at least three types of DGATs, namely two membrane-bound enzymes, DGAT1 and DGAT2 and a cytosolic enzyme, DGAT3 are functionally characterized. The acylCoA-independent reaction is catalyzed by phospholipid:diacylglycerol acyltransferase (PDAT), which use phospholipids as acyl donor and DAG as acyl acceptor to produce TAG and lysophospholipids. The Arabidopsis genome contains two close homologs to the yeast PDAT gene, but only PDAT1 activity has been confirmed by overexpression of the PDAT1 in Arabidopsis seedlings $(34 ; 164)$. All three DGATs and PDAT1 appear to be ubiquitously expressed in both vegetative and reproductive tissues of Arabidopsis $(70 ; 101 ; 110)$. While the DGAT1 transcript level is at least 4- and 10-fold higher in mature siliques and in senescing leaves, respectively, than in young leaves, the expression levels of PDAT1, DGAT2 and DGAT3 exhibit only small variations in different tissues and across various stages of leaf development (70). Among the four genes encoding TAG assembly enzymes, the transcript level of DGAT3 is consistently higher than that of the other three genes except in senescing leaves, where DGAT1 is more strongly expressed than DGAT3. Interestingly, the transcript of PDAT1 is present at higher levels than that of DGAT1 in growing and mature leaves, whereas the opposite is true in senescing leaves $(45 ; 70)$.

Recent biochemical and genetic studies in Arabidopsis have begun to shed light on the roles of DGATs and PDAT1 in TAG biosynthesis and function in vegetative plant tissues, taking advantages of tgd1 and other mutants defective in various aspects of TAG metabolism. While the role of DGAT3 may be limited to the partitioning of acyl groups to TAG during seed germination and early seedling establishment as revealed by characterizing the mutants defective in TAG breakdown (70), two other acyltransferases, PDAT1 and DGAT1 jointly play major, but distinct roles in TAG assembly in leaves, roots and floral tissues of adult plants $(44 ; 83)$. In particular, while PDAT1 appears to be more involved in TAG assembly than DGAT1 in growing leaves and young floral organs, DGAT1 is more important in TAG accumulation in senescing leaves $(44 ; 45)$, roots $(83)$ and developing seeds $(79 ; 141)$. The distinct roles of PDAT1 and DGAT1 in TAG synthesis during leaf growth and development are in line with the data from transcriptomic analyses $(45 ; 70)$. However, the acyl-CoA-dependent pathway catalyzed by DGAT1 is largely responsible for label incorporation into TAG when young Arabidopsis leaves were fed with exogenous ${ }^{14} \mathrm{C}$-labeled lauric acid (171). Other plant acyltransferases with DGAT activity include phytyl ester synthase 1 and 2 (104) and a soluble acyltransferase associated with the formation of cutins (137). The phytyl ester synthases are localized in the plastid and have been suggested to play a role in the recycling of free FAs into TAG and phytyl esters in the plastid during abiotic stress and senescence (104), thus may not contribute to cytosolic TAG synthesis in leaves. 


\section{TAG storage}

The storage of neutral lipids such as TAGs mainly involves a universal subcellular organelle named lipid droplet (LD) or oil body in the cytosol. During the past decade, studies in yeast, mammals and plant oilseeds have completely changed our perception of LD from an inert neutral lipid store to a dynamic subcellular organelle vital for lipid metabolism and homeostasis, intracellular lipid trafficking and stress responses $(18 ; 29 ; 62 ; 90 ; 114 ; 129 ; 188)$. Despite its importance, however, we have limited knowledge about the biology of LDs in vegetative tissues of plants, although its ubiquitous occurrence in leaf mesophyll cells has been known for a long time $(97 ; 163)$.

A large body of work exists on the structural and functional aspects of plant seed LDs (for reviews see $(29 ; 118 ; 155))$. Like its counterparts in other organisms, plant seed LDs are composed of a neutral lipid core coated by a monolayer of phospholipids with a specific set of hydrophobic proteins embedded. There are at least three classes of functionally characterized seed LD proteins namely oleosins, caleosins and steroleosins. Oleosins are the major class of seed-specific LD proteins that are not present in vegetative tissues of plants. Nevertheless, Arabidopsis seed oleosins can be correctly targeted to leaf LDs when transiently expressed in tobacco leaf cells $(35 ; 184)$. Ectopic expression of the Arabidopsis oleosin 1 in leaves increases leaf oil content (180; 189), reduces leaf LD size and promotes the clustering of leaf LDs (45), supporting a role of oleosins in stabilizing and regulating the size of LDs as revealed by studies in Arabidopsis seeds $(156 ; 160)$. In contrast to oleosins, caleosins and steroleosins are minor proteins in seed LDs and genes encoding caleosin homologs and steroleosins are expressed in vegetative tissues of plants, particularly under abiotic and biotic stresses $(6 ; 87 ; 157)$. A recent proteomic analysis of Arabidopsis leaf LDs identified caleosin 3 and $\alpha$-dioxygenase as their protein components involved in the generation of an antifungal compound named phytoalexin under a pathological condition, revealing a role of leaf LDs in plant defense against pathogens (157). The Arabidopsis steroleosin plays an important role in brassinosteroid biosynthesis and signaling (98) and is not targeted to leaf LDs but retained in ER when transiently expressed in leaf tobacco protoplasts (35). A new class of LD-associated proteins has recently been identified by combined lipidomic, proteomic, and transcriptomic analyses of avocado (Persea americana) mesocarp (71). This class of lipid droplet-associated proteins bears no sequence similarity to known Arabidopsis seed LD proteins and its Arabidopsis homolog was found to be associated with the surface of leaf LDs after transient expression in tobacco leaf cells (71), but its role in TAG metabolism awaits further investigation.

\section{Triacylglycerol Turnover}

\subsection{Triacylglycerol hydrolysis}


During times of carbon and energy deficiency, TAG stored in LDs is hydrolyzed to release FAs and other metabolites, and optimal utilization of this resource is vital for cellular energy balance, lipid homeostasis, membrane proliferation, cell growth and survival. The catabolism of TAG is initiated by an evolutionally conserved family of patatin domain lipases in yeast (4; 5), humans (199) and Drosophila melanogaster (61). In the model plant Arabidopsis, the sugar-dependent 1 (SDP1) encodes a TAG lipase with patatin-like acyl-hydrolase domain (40) and emerging evidence indicates that SDP1 plays a major role in TAG hydrolysis in roots and leaves of adult plants $(43 ; 83)$, in addition to young seedlings $(40 ; 81)$. There is evidence that the TAG hydrolytic activity of SDP1 is associated with LDs during postgerminative growth and early seedling establishment (40). Interestingly, it was recently showed that SDP1 is initially localized on peroxisomes and then delivered to the LD surface through peroxisome extensions during the course of seed oil mobilization (167). The subcellular localization of SDP1 in vegetative tissues of adult plants remains unknown.

Besides SDP1, there are at least two additional patatin-domain TAG lipase candidates in Arabidopsis, namely sugar-dependent1-like (SDP1L) and adipose triglyceride lipase-like (ATGLL) (40). Analysis of Arabidopsis mutants lacking SDP1 and/or SDP1L has shown that SDP1L has an overlapping role with SDP1 in TAG breakdown following seed germination (81), but this enzyme appears not to contribute significantly to TAG hydrolysis in roots and leaves of adult plants $(43 ; 83)$. In mammals, the enzymatic activity of ATGL is regulated by a coactivator protein named comparative gene identifier 58 (CGI58) (96). Although the recombinant Arabidopsis CGI58-like protein exhibited TAG lipase activity in in vitro assays (55). disruption of CGI58L or ATGLL appears to affect neither the rate of TAG mobilization in young seedlings of the mutants lacking SDP1 and SDP1L (81) nor TAG content in leaves of adult tgd1 mutant plants (43). There is evidence that loss-of-function of CGI58 in Arabidopsis leaves can result in accumulation of TAGs in cytosolic LDs (72), but this is likely mediated through the interaction of CGI58 with the Arabidopsis peroxisomal ATP binding cassette transporter1 (PXA1, also known as comatose or peroxisomal defective3) (Park et al, 2013), not via lipase activity directly. At least three other enzymes from Arabidopsis including a leaf senescence-associated protein SAG101 (68), a TAG hydrolase homologous to mammalian lysosomal acid lipase (78) and a plastid TAG lipase (130) also exhibit TAG hydrolytic activity in in vitro assays using recombinant proteins, but their roles in TAG hydrolysis in vegetative plant tissues remain to be tested.

Biochemical assays have shown that both SDP1 and SDP1L have a substrate preference for TAG over DAG and monoglycerol (MAG), implying that the patatin domain TAG lipases have to work in concert with additional lipases to completely breakdown TAG into free FAs and glycerol (40; 81). However, to date, there is little information regarding the molecular identity of lipases responsible for hydrolyzing DAG and MAG in plants. In the Arabidopsis genome, there are more than 270 genes annotated as lipases and among them 30 genes annotated as TAG or MAG lipases, but, functional characterization remains to be performed on most of these (175). 
Interestingly, a cytosolic member of the DAD1-like acyl-hydrolase family encoded by At4g18550 can specifically hydrolyze DAG and MAG at the sn-1 position and there is evidence that this protein is involved in the regulation of storage oil mobilization and early seedling establishment in Arabidopsis (84).

\subsection{FA activation, transport and $\beta$-oxidation}

Following TAG hydrolysis by lipases, FAs are activated to acyl-CoA by long-chain acyl-CoA synthetases (LACSs) prior to their entry into the peroxisome, the sole site for the breakdown of FAs via $\beta$-oxidation in plants and fungi $(56 ; 169)$. In Arabidopsis, LACSs are encoded by a family of nine genes and all but LACS5 are expressed in various tissues including leaves and roots (159). Many studies have been performed on the functional characterization of Arabidopsis LACSs $(50 ; 111 ; 150 ; 198)$, but the enzymes which activate FAs destined for peroxisomal $\beta$ oxidation in seeds and other tissues remain to be assigned though LACS6 and LACS7 are potential candidates (50). Recently, it was shown that LACS4 and LACS9 play an overlapping role in the generation of acyl-CoAs for the synthesis of galactolipids through the eukaryotic pathway in leaves (74). Whether the same two LACSs are also involved in the activation of FAs for TAG synthesis and FA breakdown in vegetative tissues requires further investigation.

The import of acyl-CoA into peroxisomes is mediated by ATP binding cassette proteins of subfamily D including PXA1 (170). The Arabidopsis PXA1 has recently been shown to possess intrinsic acyl-CoA thioesterase activity, which hydrolyzes acyl-CoAs to free FAs and coenzyme A as part of the FA transport cycle (36). Two LACS enzymes, LACS6 and LACS7 are involved in the reactivation of free FAs inside the peroxisome (50) and this reactivation requires the supply of ATP by peroxisomal adenine nucleotide translocators PNC1 and 2 (103). Disruption of PXA1 impairs TAG mobilization during early seedling establishment following germination (48; 67 ; 200) and FA turnover in leaves of adult tgd1 mutant (43) and wild-type (93) plants.

The end product of $\beta$-oxidation of FAs is acetyl-CoA, which is a key metabolite for energy production via mitochondrial respiration and for the synthesis of carbon skeletons via the glyoxylate cycle and gluconeogenesis. While the role of glyoxylate cycle in the bulk conversion of oils into carbohydrates has been well established $(56 ; 169)$, two marker enzyme of the glyoxylate cycle, namely malate synthase and isocitrate lyase are not expressed in mature Arabidopsis plants $(24 ; 179)$. Therefore, in contrast to young seedlings, where $\beta$-oxidation of FAs supplies energy and carbohydrates, in mature leaves under most conditions the glyoxylate cycle activity appears to be absent and $\beta$-oxidation supplies energy but not carbohydrates (93). However, the activities of malate synthase and isocitrate lyase are induced in leaves of barley, spinach and cucumber during senescence $(57 ; 63 ; 95)$, supporting a role for the glyoxylate cycle and gluconeogenesis in senescence-related processes (175). 


\section{TAG metabolism and lipid homeostasis}

Under conditions of lipid overload, excess FAs are converted into biologically inert neutral lipid in the form of TAG which is packaged in LDs, thereby sequestering them away from cellular membranes. Cells deficient in TAG synthesis show increased levels of membrane lipids, and accumulate toxic lipid intermediates such as DAG and free FAs. They also exhibit, massive proliferation of intracellular membranes and ultimately undergo programmed cell death (89). In plants, PDAT1 and DGAT1 mediated TAG synthesis is essential for pollen and embryo development (196), and deficiency of DGAT1 activity has negative effects on overall growth and developmental $(79 ; 107 ; 162)$. In the tgd1 mutant, disruption of PDAT1 causes premature cell death in growing leaves and floral organs with concomitant increases in the levels of free FAs, DAG and membrane phospholipids along with severe decreases in TAG levels (44). Thus, the role of TAG synthesis in lipid homeostasis and cell survival is evolutionarily conserved in eukaryotes ranging from yeast and plants to humans.

TAG metabolism encompasses multiple subcellular organelles, which necessitates extensive trafficking of FAs and other intermediates between cellular organelles and within and across biological membranes. During FA trafficking, special cellular mechanisms appear to exist to strictly limit the buildup of toxic FAs in the cytosol. One example is the PDAT1/LPCAT cycle involved in TAG synthesis in growing leaves (44) (Figure 4). In this cycle, nascent FAs exported from the plastid are first incorporated into PC $(12)$, catalyzed by LPCATs $(11 ; 185)$, likely at the chloroplast envelope membranes $(19 ; 88 ; 173)$. PC is used for DAG esterification to produce TAG catalyzed by PDAT1 in the ER (34). The by-product of this reaction, lysoPC, can rapidly partition from the ER to the chloroplast (19), where it is re-acylated to produce PC $(19 ; 88 ; 173)$, the substrate for PDAT1-mediated TAG synthesis. In principle, the PDAT1/LPCAT cycle can be more efficient in protecting against FA-induced toxicity if it occurs at membrane contact sites between the ER and the plastid $(20 ; 186)$, as such sites could enable ER-residing PDAT1 to access PC in the plastid envelope for DAG esterification and/or plastidic PC to be effectively translocated to ER for PDAT1-mediated TAG synthesis, thereby avoiding overabundance of toxic free acyl groups in the cytosol.

Recent evidence indicates that LDs serve as a conduit for FA breakdown and efficient $\beta$ oxidation requires close association of LDs with organelles carrying out $\beta$-oxidation to facilitate the intraorganelle movement of FAs. Unlike plants and fungi, $\beta$-oxidation of FAs in mammals occurs in the mitochondria. Under nutrient starvation, an important source of FAs for $\beta$-oxidation is derived from autophagic digestion of membrane structures (161). Rather than being directly imported into mitochondria for energy production, FAs derived from membrane lipids are first incorporated into TAG and stored in LDs prior to being released by TAG lipases before entering the mitochondrial $\beta$-oxidation pathway (136). During TAG consumption, the trafficking of FAs from LDs into mitochondria requires the close association of the two organelles (7) and the establishment of mitochondrial networks through fusion (136). In plants, the lipin homologs, in addition to PDAT1, SDP1 and PXA1 direct de novo-synthesized FAs towards peroxisomal $\beta$ - 
oxidation through a transient TAG pool and this process is important for maintaining membrane lipid homeostasis in tgd1 leaves (43) (Figure 4). Transfer of FAs released from LDs into peroxisomes appears to require close physical apposition of these two organelles in plants also (167; 169).

\section{Strategies for Engineering Oil Accumulation in Vegetative Tissues}

Metabolic engineering strategies to enhance oil accumulation in plants include increasing the supply of FAs, increasing TAG assembly activities and blocking TAG breakdown pathways, as recently reviewed $(26 ; 30 ; 38 ; 121 ; 127 ; 175 ; 182)$. A list of genes, manipulation of which resulting in increased TAG content in vegetative plant tissues was recently presented (182). A difficulty in comparing reports on increasing TAG accumulation is that results are often cited as fold-increases. Because very low levels of TAG accumulate in most wild type leaves, and low levels of TAG are difficult to quantitate, comparisons based on \% of DW are far more useful and reliable (193).

Theoretically, increasing the supply of FAs for TAG synthesis can be achieved by 1) increasing the flux of carbon into FA synthesis; 2) overexpressing transcriptional or other regulators controlling FA synthesis 3) increasing the expression of ACCase, the first committed enzyme in the plastidic FA synthesis pathway; 4) suppressing competing pathways for FA utilization including membrane lipid synthesis; and 5) increasing carbon partitioning into FA synthesis by blocking starch synthesis. Petrie and colleagues have categorized three aspects to increasing vegetative TAG, namely push, by modulating WRI1, other transcription factors, ACCase or mutants in TGD1 or MGD1, pull by acyltransferases, and protection by oleosin, LEC1,2 and mutants of SDP1 and CGI58 (182). Because modulation of the push factors on their own don't increase TAG accumulation to a significant degree, possibly because WRI1 induces futile cycling by upregulating both fatty acid synthesis and fatty acid degradation (60), it might be more useful to think of these factors in terms of defining capacity to increase TAG accumulation. In contrast, in the absence of push factor modulation, overexpression of acyltransferases increase sink strength (23) and combinations of pull and protect such as coexpression of DGAT1 with cys-OLEOSIN appear to dramatically increases sink strength to the point that along with TAG increase, photosynthesis and even biomass are seen to increase (189). This is a dramatic effect considering that lipid synthesis is highly demanding both in terms of energy and reducing equivalents (Figure 2). While leaves have evolved as source tissues, being the site of photosynthesis provides access to an abundant local source of energy and reducing equivalents.

\subsection{Increasing the flux of carbon into FA synthesis}

Attempts to increase the supply of precursors for FA synthesis by creating simple changes to central metabolism have generally failed $(37 ; 168)$. The lack of success likely results from the fact that individual enzymes rarely determine the flux though a pathway, rather flux control is 
often shared amongst multiple components of the pathway (117). However, beyond the classical concepts of flux control, allosteric control of enzyme activity can be substantially involved in control of carbon partitioning. Quantitative multilevel analysis of central metabolism in developing Brassica napus embryos from a series of Brassica lines that exhibit a range of starch:lipid ratios implicated pyruvate kinase, the enzyme responsible for pyruvate production as contributing to flux control over the production of pyruvate for oil biosynthesis (152). Flux control was proposed to be mediated by concerted allosteric feedback control of phosphofructokinase and ADP-glucose pyrophosphorylasel (ADG1) by PEP and 3phosphoglycerate (3PGA), respectively.

\subsection{Increasing FA supply}

Because FA synthesis in seeds is subject to transcriptional regulation, a direct approach to increasing TAG synthesis in vegetative tissue is to initiate the seed oil synthesis program by ectopically expressing transcription factors. In seeds, TAG deposition is under developmental control that initiates at the completion of seed morphogenesis when maturation begins (146). It is principally under the control of a set of B3-domain containing master transcriptional activating factors referred to as AFL after their three members: ABSCISIC ACID INSENSITIVE1, FUSCA3 and LEAFY COTYLEDON2 (LEC2). AFL activators have redundant functions and act in concert with LEC1 to activate the maturation program. The maturation program can be repressed by chromatin remodeling factors such as PICKLE (124), or a set of three B3-like proteins that compete for AFL binding (16). Ectopic expression of LEC2 was found to be sufficient to trigger the accumulation of oil and seed specific mRNAs in the leaves of Arabidopsis (148). The action of LEC2 (and possibly LEC1) on lipid synthesis is specified by WRI1 (Figure 3). As discussed in section 2.2, the influence of WRI1 is more specific to the induction of genes associated with FA synthesis and glycolysis (and SUC2) and its effects are potentiated by sugar $(115 ; 144)$. Whether sugar potentiation is related solely to the availability of carbon, or perhaps more likely, involves interplay between oil- and sugar signaling networks (140) remains to be determined. The overexpression of LEC2 induces both WRI1 and oil droplet-specific proteins such as oleosins (148), however, expression of LEC2 can produce strong negative pleiotropic developmental relative to those reported for the expression of WRI1 $(85 ; 144 ; 166)$. The use of chemical-, or senescence-inducible promoters can mitigate some of the negative pleiotropic effects $(3 ; 86 ; 148)$. In terms of TAG accumulation, expression of WRI1 on its own led to a 2.8-fold increase in TAG in whole plant tissue (144), whereas expression of LEC2 led to the accumulation of $0.8 \%$ DW in senescing Arabidopsis leaves (162) and a more than doubling of total lipids in tobacco to $6.8 \% \mathrm{DW}(3)$. The effects of ectopic expression of WRI1's from several sources highlighted a range of TAG accumulation when transiently expressed in $N$. benthamiana leaves (60). 
Early attempts to increasing FA synthesis focused on potential rate-limiting steps in the FA synthesis pathway with mixed results. For example, coordinate overexpression of all four heteromeric ACCase subunits in cyanobacteria increased FA biosynthesis (106), whereas in Arabidopsis, expression of BCCP resulted in inactivation of the plastid ACCase and reduced seed oil content (168). Overexpression of 3-ketoacyl-acyl-carrier protein synthase III (KAS III) notably reduced the rate of FA synthesis in developing oilseeds (37). In Brassica, plastidial targeting of the Arabidopsis homomeric ACCase in Brassica seeds resulted in an approximately $5 \%$ increase in seed TAG under growth chamber conditions (139). It should be noted that oil content is dependent on light availability and other factors(102), and so growth chamber and field results can differ substantially.

\subsection{Disruption of pathways competing with TAG synthesis}

Silencing of monogalactosyldiacylglycerol synthase (MGD1), the first committed step in thylakoid galactolipid synthesis resulted in a decrease in galactolipid content and an 8.3-fold increase in TAG accumulation in tobacco leaves (190). Perhaps surprisingly, disruption of starch synthesis produced only minor increases in TAG accumulation. When APS1, a major isoform of the small subunit of ADP-glucose pyrophosphorylase involved in starch biosynthesis was downregulated, by RNAi, the resulting lines accumulated more hexoses, less starch but only 30$40 \%$ more TAG compared to WT (144).

\subsection{Increasing TAG assembly activities}

As discussed in a recent review (Morandini, 2013), flux control of many metabolic pathways that supply building blocks for the synthesis of macromolecules including lipids, proteins, nucleic acids and carbohydrates often occurs on the demand side. Therefore, increasing the demand for precursors can be more effective at enhancing flux through many pathways than increasing the supply. This strategy has been referred to as pull with respect to TAG assembly (182). In the case of the engineering of TAG accumulation, overexpression of TAG assembly enzymes appears to be more successful at boosting TAG content in seeds (Jako et al., 2001(105)) as well as vegetative tissues (Fan et al., 2013b) than overexpression of enzymes mediating FA synthesis (Roesler et al., 1997; Dehesh et al., 2001; Thelen and Ohlrogge, 2002).

DGAT has long been recognized as an activity for which ectopic expression can cause substantial increases in the accumulation of vegetative TAG (105). Expression of the Arabidopsis DGAT1 in tobacco yielded between a 7 - and 20-fold increase in TAG $(3 ; 23)$, whereas expression of a Chlamydomonas DGAT2 increased TAG accumulation in Arabidopsis by 25-fold and changed the TAG composition by favoring the incorporation of very-long-chain FAs (145). Recent analysis showed that the addition of an N-terminal peptide tag could mask 
the deleterious influence of B. napus DGAT1's N-terminal sequence, stabilizing it and increasing its accumulation in yeast, and increasing its in vitro activity by a remarkable 150-fold (58). Intriguingly, overexpression of PDAT1 in Arabidopsis enhanced the rate of FA synthesis, leading to the accumulation of $2.6 \% \mathrm{DW}$ of TAG in leaves (45). The heterologous expression of mouse monoacylglycerol (MAG) acyltransferases 1 and 2 in tobacco led to 7-9-fold increases in the accumulation of TAG by a Kennedy pathway-independent mechanism involving the conversion of glycerol-3-phosphate to MAG by sn-2 glycerol-3-phosphate:acyl-CoA acyltransferase (GPAT) and on to TAG by the action of MGAT (133).

Recently, SEIPIN, a protein that localizes to the ER of mammals and regulates adipocyte differentiation, lipolysis and determine the size and distribution of lipid droplets (Fei et al., 2011) was identified in plants. Overexpression of SEIPIN1 in Arabidopsis increased numbers of LDs and TAG content in leaves and boosted oil content in seeds (25).

\subsection{Blocking TAG breakdown pathways}

Stabilizing oil storage compartments by overexpressing oleosins is an effective means to boosting TAG accumulation in vegetative plant tissues. Oleosins are small amphipathic proteins that intercalate with TAG and form an interface with the cytoplasm. They also limit the size of LDs and appear to serve as a steric barrier protecting TAG from lipase activities $(155 ; 160)$. Support for the notion that oleosins protect TAG comes from the expression in Arabidopsis of poly-oleosins comprising up to six head-to-tail oleosin repeats that was shown to increase the structural rigidity of the oil bodies (153). In other work, oleosins were engineered to contain multiple cysteine residues (Cys-oleosins) that form intermolecular disulfide bonds which form a cross-linked network (189).

As an alternative approach to physically protecting the TAG in LD is to disrupt TAG lipase activity or the transport of lipids into the peroxisome by disruption of SDP1 or PXA1. Disruption of SDP1 resulted in increases in TAG accumulation of close to $1 \%$ of DW in roots with smaller effects seen in other tissues (82). Disruption of PXA1 results in the accumulation of up to $1.8 \%$ TAG in senescing leaves (162). Another potential target to block FA turnover is the 3-ketoacylCoA thiolase (KAT) (54), a key enzyme in $\beta$-oxidation. The kat2 null mutant is viable if germinated in the presence of sugar, however, it exhibits slower and more stunted growth than pxa1. The Arabidopsis homolog of CGI-58 was identified by Chapman's group (72), mutations of which in humans result in hyperaccumulation of lipid droplets in tissues that do not normally store lipids (192). Plants containing mutated CGI-58 exhibit a 10-fold increase in TAG in mature leaves with minimal detrimental phenotypic effects (72), suggesting that plant and mammalian CGI-58 proteins share a common function, possibly via interaction with PXA1 in plants (131). 


\subsection{Combinatorial approaches}

As discussed above, many individual factors have been shown to increase the accumulation of TAG to several percent of DW. Because flux control is shared amongst different pathway steps, and biochemical feedback control tends to buffer against single-gene changes, the most effective strategies to substantially increase TAG accumulation typically involve simultaneous downregulation, up-regulation, or heterologous expression of two or more factors. Indeed many successful modulations of two- or more genes have been reported. For example, co-expression of Cys-oleosins along with DGAT in Arabidopsis resulted in increased biomass, photosynthetic rate and the doubling of FA content in mature leaves, that was attributed to the protection of TAG within LD from degradation (189). Coexpression of DGAT and WRI1 produced a synnergisitc increase in TAG accumulation when transiently coexpressed in $N$. benthamiana (181). Their stable expression in Arabidopsis yielded TAG accumulation in the 2-3\% of DW range albeit with significant reduction in total biomass (83). Coexpression of DGAT1 and WRI1 in the sdp1 mutant background approximately doubled the TAG accumulation in Arabidopsis (83); however, further enhancements were not seen upon expression in sdp1 cgi-58, or sdp1 pxa1, presumably because mutations in the same pathway were not additive. In contrast, when WRI1 was overexpressed in a line deficient in starch biosynthesis, a 5.8-fold increase in TAG accumulation was observed relative to the parental line in which starch was reduced to half of that of wild type (144). Overexpressing Arabidopsis PDAT1, along with Arabidopsis OLEOSIN1 in the tgd1 mutant background resulted in leaf TAG accumulation of $8.6 \%$ with total FAs accumulating to $16.2 \%$ (45). Further experiments combining tgd1 with sdp1 or pxa1 resulted in approximately 9\% TAG accumulation in Arabidopsis leaves (43). Recently Petrie and colleagues reported the accumulation of $>15 \%$ TAG (17.7\% total lipids) by DW in stably transformed Nicotiana tabacum leaves coexpressing the Arabidopsis WRI1, and DGAT1 along with the sesame OLEOSIN driven by the Arabidopsis RuBisCo small subunit promoter (for WRI1 and OLEOSIN) and 35S promoter (for DGAT1). The authors report that the accumulation of TAG at levels close to those of soybean seeds was without detrimental effects on plant development and seed viability similar to what was observed upon the overexpression of cysOle1 and DGAT in Arabidopsis (189). This raises the question as to what proportion of the DW of vegetative tissues TAG can be reached before significant detrimental effects become inevitable. The answer to this question is likely to depend on the timing of accumulation, the plant species, and the tissue (leaves vs stems). A lack of negative consequences on source tissues such as leaves may be aided by the inert nature of TAG based on its immiscibility with the aqueous cytoplasmic phase, and its physical sequestration in LD. We note the currently most successful three-gene over-expression strategy (180) and the two 2-gene disruption strategies (43) contain non-overlapping gene sets. It is therefore conceivable that further combinations of these factors alone, or in combination with other factors, modulations of which have been shown

to result in enhancement of vegetative TAG accumulation described above, will likely yield further increases in TAG accumulation. 


\section{Opportunities and Challenges}

American founding father Thomas Jefferson wrote: "The greatest service which can be rendered any country is to add a useful plant to its culture". He went on to exemplify grain and oil as examples (73). Recent discoveries regarding the biochemistry and regulation of vegetative oil metabolism are fueling optimism that novel vegetative-oil accumulating crops may soon be added to our culture. The incorporation of enzyme variants (58) or perhaps transcription factor variants with enhanced activity/stability (112) presents a particularly promising approach to increasing oil yield. The discovery that several combinations of gene mutations can lead to TAG accumulation of close to $10 \%$ in vegetative tissue without the overexpression of transgenes (43) raises an intriguing possibility of creating vegetative TAG-accumulating crops by targeted disruption of tissue-specific isoforms of these, and maybe other genes, using CRISPR-CAS9 gene editing or equivalent technologies (21). Conceivably mutation-based strategies could lead to non-GM crops that streamline commercialization. Other promising enabling technologies to identify targets for TAG accumulation include metabolic flux analysis to quantify fluxes through central metabolism (123) and the lipid biosynthetic network (10) of developing seeds. Also, predictive in silico modeling approaches (151) might be refined to select non-intuitive combinations of potential targets to increase oil accumulation.

However, many questions remain to be answered including: Which combinations of TAGaccumulating genes will provide optimal oil accumulation while minimizing yield drag and preserving coproduct yields? Which promoters will facilitate optimal TAG accumulation in the desired tissues at the desired developmental stage? Will vegetative oilcrops be more susceptible to pathogens or herbivory or adverse environmental conditions? If so, can mitigation strategies be identified? Will results obtained in growth chambers or greenhouses be observed under field conditions? Because most work in this area has focused on model plants, perhaps the major open question is: Which crop plants should be targeted to accumulate vegetative TAG? Candidates should have sufficient biomass yield and geographical range to produce large quantities of oil, be able to thrive on marginal land and be productive with mammal agricultural inputs. Sugarcane represents one potential candidate, which accumulates excess photosynthate as sugar to approximately $50 \%$ of its stem biomass by DW that could potentially be converted to TAG (195). Because of its exceptional biomass yield, "oilcane" i.e., an engineered sugarcane in which a significant fraction of its stored sugar is converted to TAG could provide a meaningful contribution to our liquid transportation fuel needs. Successful strategies, initially developed for sugarcane could in theory be transferred to its more cold-tolerant relative sweet sorghum or eventually to perennial crops such as Miscanthus or poplar which require low inputs. Sugarcane and sorghum are also appealing because there is an existing infrastructure to grow and harvest sugarcane and sorghum and harvest oil which can be converted to biodiesel in a single unit operation. In terms of energy yield, achieving vegetative TAG accumulation to $10 \%$ of DW increases the energy content of the crop by $30 \%$ relative to fermentation to ethanol (127). 
While vegetative TAG can be viewed as a future source for biofuel uses, the technology could be equally well be combined with other successful strategies for modifying the composition of oil such as accumulating nutritionally valuable lipids (142) or industrially useful feedstocks (120) as recently demonstrated for medium chain fatty acid accumulation in leaves (138).

\section{Summary points}

1. Although plant vegetative tissues such as leaves possess a high inherent capacity to synthesize, store and mobilize TAG, they normally do not accumulate TAG. This is because leaves evolved as source tissues highly specialized for photosynthetic carbohydrate synthesis and export and the majority of FAs and DAG are used for the synthesis of photosynthetic membranes.

2. The activity of the plastidial ACCase, which catalyzes the first committed step in FA synthesis, can potentially be modulated by a wide array of biochemical, transcriptional and posttranslational mechanisms.

3. The lipin family of PA phosphohydrolases play important roles in regulating FA and DAG partitioning between TAG and membrane lipid synthesis in leaves via modulation of lipid PA levels.

4. TAG assembly in vegetative tissues is mediated by acyl-CoA-dependent- and acyl-CoAindependent mechanisms. In Arabidopsis, PDAT1 and DGAT1 play major, but distinct roles in TAG accumulation in leaves.

6. TAG is a key intermediate in fatty acid breakdown through the peroxisomal $\beta$-oxidation pathway which is vital for protection against fatty acid-induced cell death in leaves under conditions of fatty acid overload.

7. The most effective strategies to substantially increase TAG accumulation involve simultaneous down-regulation, up-regulation, or heterologous expression of two or more factors.

Acknowledgements: We acknowledge the support of the Division of Chemical Sciences, Geosciences, and Biosciences, Office of Basic Energy Sciences of the U.S. Department of Energy (grant no. DOE KC0304000). We thank John Ohlrogge, Kent Chapman and Jorg Schwender and for critical reading of the manuscript.

\section{Figure legend}


Figure 1. Overview of the source and sink relationship in oilseed plants. Abbreviations as defined in the text; except AA, amino acids.

Figure 2. Overview of carbon flux in relation to FA and sucrose synthesis in a leaf mesophyll cell. Photosynthetic light reactions generate ATP and DNAPH, which power both carbon fixation via the Calvin cycle and FA synthesis catalyzed by ACCase and a series of enzymatic reactions collectively referred to as fatty acid synthase (FAS). Dotted arrows indicate multiple reactions. Red arrows indicate steps in glycolysis. Green letters indicate proteins. Abbreviations as defined in the text; except: ADPGlc, ADP_glucose; Glc1P, glucose-1-phosphate; Glc6P, glucose-6-phosphate; MEX1, maltose excess protein1; pGlcT, plastidial glucose transporter; 2PGA, 2-phosphoglycerate.

Figure 3. Scheme of factors that can potentially regulate FA synthesis. Abbreviations as defined in the text

Figure 4. Overview of TAG metabolism pathways in a leaf mesophyll cell. Abbreviations as defined in the text.

\section{References}

1. Adeyo O, Horn PJ, Lee SK, Binns DD, Chandrahas A, et al. 2011. The yeast lipin orthologue Pah1p is important for biogenesis of lipid droplets. J. Cell Biol. 192:1043-55

2. Andre C, Haslam RP, Shanklin J. 2012. Feedback regulation of plastidic acetyl-CoA carboxylase by 18:1-acyl carrier protein in Brassica napus. PNAS 109:10107-12 *Identified biochemical feedback for plastidial ACCase.

3. Andrianov V, Borisjuk N, Pogrebnyak N, Brinker A, Dixon J, et al. 2010. Tobacco as a production platform for biofuel: overexpression of Arabidopsis DGAT and LEC2 genes increases accumulation and shifts the composition of lipids in green biomass. Plant Biotechnol. J. 8:277-87 *Demonstrates synergestic effects of expressing DGAT and LEC2 on oil accumulation in tobacco leaves.

4. Athenstaedt K, Daum G. 2003. YMR313c/TGL3 encodes a novel triacylglycerol lipase located in lipid particles of Saccharomyces cerevisiae. J. Biol. Chem. 278:23317-23

5. Athenstaedt K, Daum G. 2005. Tgl4p and Tgl5p, two triacylglycerol lipases of the yeast Saccharomyces cerevisiae are localized to lipid particles. J. Biol. Chem. 280:37301-9

6. Aubert Y, Vile D, Pervent M, Aldon D, Ranty B, et al. 2010. RD20, a stress-inducible caleosin, participates in stomatal control, transpiration and drought tolerance in Arabidopsis thaliana. Plant Cell Physiol. 51:1975-87

7. Barbosa AD, Savage DB, Siniossoglou S. 2015. Lipid droplet-organelle interactions: emerging roles in lipid metabolism. Curr. Opin. Cell Biol. 35:91-7

8. Barthole G, Lepiniec L, Rogowsky PM, Baud S. 2012. Controlling lipid accumulation in cereal grains. Plant Sci. 185-186:33-9

9. Bates PD, Browse J. 2011. The pathway of triacylglycerol synthesis through phosphatidylcholine in Arabidopsis produces a bottleneck for the accumulation of unusual fatty acids in transgenic seeds. Plant J. 68:387-99 
10. Bates PD, Durrett TP, Ohlrogge JB, Pollard M. 2009. Analysis of acyl fluxes through multiple pathways of triacylglycerol synthesis in developing soybean embryos. Plant physiol. 150:55-72

11. Bates PD, Fatihi A, Snapp AR, Carlsson AS, Browse J, Lu CF. 2012. Acyl editing and headgroup exchange are the major mechanisms that direct polyunsaturated fatty acid flux into triacylglycerols. Plant physiol. 160:1530-9

12. Bates PD, Ohlrogge JB, Pollard M. 2007. Incorporation of newly synthesized fatty acids into cytosolic glycerolipids in pea leaves occurs via acyl editing. J. Biol. Chem. 282:31206-16 *Indentified PC acyl editing as an important component of glycerolipid metabolism.

13. Bates PD, Stymne S, Ohlrogge J. 2013. Biochemical pathways in seed oil synthesis. Curr. Opin. Plant Biol. 16:358-64

14. Baud S, Bourrellier ABF, Azzopardi M, Berger A, Dechorgnat J, et al. 2010. PII is induced by WRINKLED1 and fine-tunes fatty acid composition in seeds of Arabidopsis thaliana. Plant J. 64:291-303

15. Baud S, Lepiniec L. 2009. Regulation of de novo fatty acid synthesis in maturing oilseeds of Arabidopsis. Plant Physiol. Biochem. 47:448-55

16. Baud S, Lepiniec L. 2010. Physiological and developmental regulation of seed oil production. Prog. Lipid Res. 49:235-49

17. Baud S, Mendoza MS, To A, Harscoet E, Lepiniec L, Dubreucq B. 2007. WRINKLED1 specifies the regulatory action of LEAFY COTYLEDON2 towards fatty acid metabolism during seed maturation in Arabidopsis. Plant J. 50:825-38

18. Beller M, Sztalryd C, Southall N, Bell M, Jackle H, et al. 2008. COPI complex is a regulator of lipid homeostasis. Plos Biol. 6:e292

19. Bessoule JJ, Testet E, Cassagne C. 1995. Synthesis of phosphatidylcholine in the chloroplast envelope after import of lysophosphatidylcholine from endoplasmic-reticulum membranes. Eur.J. Biochem. 228:490-7

20. Block MA, Jouhet J. 2015. Lipid trafficking at endoplasmic reticulum-chloroplast membrane contact sites. Curr. Opin. Cell Biol. 35:21-9

21. Bortesi L, Fischer R. 2015. The CRISPR/Cas9 system for plant genome editing and beyond. Biotechnol. Adv. 33:41-52

22. Bourrellier ABF, Valot B, Guillot A, Ambard-Bretteville F, Vidal J, Hodges M. 2010. Chloroplast acetyl-CoA carboxylase activity is 2-oxoglutarate-regulated by interaction of PII with the biotin carboxyl carrier subunit. PNAS 107:502-7

23. Bouvier-Nave P, Benveniste P, Noiriel A, Schaller H. 2000. Expression in yeast of an acylCoA:diacylglycerol acyltransferase cDNA from Caenorhabditis elegans. Biochem. So.c Trans. 28:692-5

24. Buchanan-Wollaston V, Page T, Harrison E, Breeze E, Lim PO, et al. 2005. Comparative transcriptome analysis reveals significant differences in gene expression and signalling pathways between developmental and dark/starvation-induced senescence in Arabidopsis. Plant J. 42:567-85

25. Cai Y, Goodman JM, Pyc M, Mullen PT, Dyer JM, Chapman KD. 2015. Arabidopsis SEIPIN proteins modulate triacylglycerol accumulation and influence lipid droplet proliferation. Plant Cell In press. *Demonstrates a role for SEIPIN in TAG accumulation in plants.

26. Carlsson AS, Yilmaz JL, Green AG, Stymne S, Hofvander P. 2011. Replacing fossil oil with fresh oil - with what and for what? Eur. J. Lipid Sci. Tech. 113:812-31

27. Carrasco S, Merida I. 2007. Diacylglycerol, when simplicity becomes complex. Trends Biochem. Sci. 32:27-36

28. Cernac A, Benning C. 2004. WRINKLED1 encodes an AP2/EREB domain protein involved in the control of storage compound biosynthesis in Arabidopsis. Plant J. 40:575-85 
29. Chapman KD, Dyer JM, Mullen RT. 2012. Biogenesis and functions of lipid droplets in plants. J. Lipid Res. 53:215-26

30. Chapman KD, Dyer JM, Mullen RT. 2013. Commentary: Why don't plant leaves get fat? Plant Sci. 207:128-34

31. Chapman KD, Ohlrogge JB. 2012. Compartmentation of triacylglycerol accumulation in plants. J. Biol. Chem. 287:2288-94

32. Chen M, Mooney BP, Hajduch M, Joshi T, Zhou M, et al. 2009. System analysis of an Arabidopsis mutant altered in de novo fatty acid synthesis reveals diverse changes in seed composition and metabolism. Plant Physiol. 150:27-41

33. Craddock CP, Adams N, Bryant FM, Kurup S, Eastmond PJ. 2015. PHOSPHATIDIC ACID PHOSPHOHYDROLASE regulates phosphatidylcholine biosynthesis in Arabidopsis by phosphatidic acid-mediated activation of CTP:PHOSPHOCHOLINE CYTIDYLYLTRANSFERASE activity. Plant Cell 27:1251-64

34. Dahlqvist $A$, Stahl U, Lenman M, Banas A, Lee $M$, et al. 2000. Phospholipid:diacylglycerol acyltransferase: an enzyme that catalyzes the acyl-CoA-independent formation of triacylglycerol in yeast and plants. PNAS 97:6487-92

35. De Domenico S, Bonsegna S, Lenucci MS, Poltronieri P, Di Sansebastiano GP, Santino A. 2011. Localization of seed oil body proteins in tobacco protoplasts reveals specific mechanisms of protein targeting to leaf lipid droplets. J. Integ. Plant Biol. 53:858-68

36. De Marcos Lousa C, van Roermund CW, Postis VL, Dietrich D, Kerr ID, et al. 2013. Intrinsic acylCoA thioesterase activity of a peroxisomal ATP binding cassette transporter is required for transport and metabolism of fatty acids. PNAS 110:1279-84

37. Dehesh K, Tai H, Edwards P, Byrne J, Jaworski JG. 2001. Overexpression of 3-ketoacyl-acyl-carrier protein synthase IIIs in plants reduces the rate of lipid synthesis. Plant Physiol. 125:1103-14

38. Durrett TP, Benning C, Ohlrogge J. 2008. Plant triacylglycerols as feedstocks for the production of biofuels. Plant J. 54:593-607

39. Dwyer JR, Donkor J, Zhang P, Csaki LS, Vergnes L, et al. 2012. Mouse lipin-1 and lipin-2 cooperate to maintain glycerolipid homeostasis in liver and aging cerebellum. PNAS 109:E2486-95

40. Eastmond PJ. 2006. SUGAR-DEPENDENT1 encodes a patatin domain triacylglycerol lipase that initiates storage oil breakdown in germinating Arabidopsis seeds. Plant Cell 18:665-75

41. Eastmond PJ, Quettier AL, Kroon JTM, Craddock C, Adams N, Slabas AR. 2010. PHOSPHATIDIC ACID PHOSPHOHYDROLASE1 and 2 regulate phospholipid synthesis at the endoplasmic reticulum in Arabidopsis. Plant Cell 22:2796-811

42. Eccleston VS, Ohlrogge JB. 1998. Expression of lauroyl-acyl carrier protein thioesterase in Brassica napus seeds induces pathways for both fatty acid oxidation and biosynthesis and implies a set point for triacylglycerol accumulation. Plant Cell 10:613-21

43. Fan J, Yan C, Roston R, Shanklin J, Xu C. 2014. Arabidopsis Lipins, PDAT1 Acyltransferase, and SDP1 Triacylglycerol Lipase Synergistically Direct Fatty Acids toward beta-Oxidation, Thereby Maintaining Membrane Lipid Homeostasis. Plant Cell 26, 4119-34 *Identified TAG as an intermediate in fatty acid breakdown in leaves.

44. Fan J, Yan C, Xu C. 2013. Phospholipid:diacylglycerol acyltransferase-mediated triacylglycerol biosynthesis is crucial for protection against fatty acid-induced cell death in growing tissues of Arabidopsis. Plant J. 76:930-42

45. Fan J, Yan C, Zhang X, Xu C. 2013. Dual role for phospholipid:diacylglycerol acyltransferase: enhancing fatty acid synthesis and diverting fatty acids from membrane lipids to triacylglycerol in Arabidopsis leaves. Plant Cell 25:3506-18

46. Flugge UI, Hausler RE, Ludewig F, Gierth M. 2011. The role of transporters in supplying energy to plant plastids. J. Exp. Bot. 62:2381-92 
47. Focks N, Benning C. 1998. wrinkled1: A novel, low-seed-oil mutant of Arabidopsis with a deficiency in the seed-specific regulation of carbohydrate metabolism. Plant physiol. 118:91-101

48. Footitt S, Slocombe SP, Larner V, Kurup S, Wu Y, et al. 2002. Control of germination and lipid mobilization by COMATOSE, the Arabidopsis homologue of human ALDP. EMBOJ. 21:2912-22

49. Fukuda N, Ikawa Y, Aoyagi T, Kozaki A. 2013. Expression of the genes coding for plastidic acetylCoA carboxylase subunits is regulated by a location-sensitive transcription factor binding site. Plant Mol. Biol. 82:473-83

50. Fulda M, Shockey J, Werber M, Wolter FP, Heinz E. 2002. Two long-chain acyl-CoA synthetases from Arabidopsis thaliana involved in peroxisomal fatty acid beta-oxidation. Plant J. 32:93-103

51. Furumoto T, Yamaguchi T, Ohshima-Ichie Y, Nakamura M, Tsuchida-Iwata Y, et al. 2011. A plastidial sodium-dependent pyruvate transporter. Nature 476:472-5

52. Geigenberger P. 2011. Regulation of starch biosynthesis in response to a fluctuating environment. Plant Physiol. 155:1566-77

53. Geigenberger $P$, Kolbe A, Tiessen A. 2005. Redox regulation of carbon storage and partitioning in response to light and sugars. J. Exp. Bot. 56:1469-79

54. Germain V, Rylott EL, Larson TR, Sherson SM, Bechtold N, et al. 2001. Requirement for 3ketoacyl-CoA thiolase-2 in peroxisome development, fatty acid beta-oxidation and breakdown of triacylglycerol in lipid bodies of Arabidopsis seedlings. Plant J. 28:1-12

55. Ghosh AK, Chauhan N, Rajakumari S, Daum G, Rajasekharan R. 2009. At4g24160, a Soluble AcylCoenzyme A-Dependent Lysophosphatidic Acid Acyltransferase. Plant Physiol. 151:869-81

56. Graham IA. 2008. Seed storage oil mobilization. Annu. Rev. Plant Biol. 59:115-42

57. Graham IA, Leaver CJ, Smith SM. 1992. Induction of malate synthase gene expression in senescent and detached organs of cucumber. Plant Cell 4:349-57

58. Greer MS, Truksa M, Deng W, Lung SC, Chen G, Weselake RJ. 2015. Engineering increased triacylglycerol accumulation in Saccharomyces cerevisiae using a modified type 1 plant diacylglycerol acyltransferase. Appl. Microbiol. Biotech. 99:2243-53

59. Grennan AK. 2006. Regulation of starch metabolism in Arabidopsis leaves. Plant Physiol. 142:1343-5

60. Grimberg A, Carlsson AS, Marttila S, Bhalerao R, Hofvander P. 2015. Transcriptional transitions in Nicotiana benthamiana leaves upon induction of oil synthesis by WRINKLED1 homologs from diverse species and tissues. BMC Plant Biol. 15:192 *Identified WRI1-induced transcripts in leaf tissue.

61. Gronke S, Mildner A, Fellert S, Tennagels N, Petry S, et al. 2005. Brummer lipase is an evolutionary conserved fat storage regulator in Drosophila. Cell Metab. 1:323-30

62. Guo Y, Walther TC, Rao M, Stuurman N, Goshima G, et al. 2008. Functional genomic screen reveals genes involved in lipid-droplet formation and utilization. Nature 453:657-61

63. Gut H, Matile P. 1988. Apparent induction of key enzymes of the glyoxylic-acid cycle in senescent barley leaves. Planta 176:548-50

64. Han GS, Siniossoglou S, Carman GM. 2007. The cellular functions of the yeast lipin homolog PAH1p are dependent on its phosphatidate phosphatase activity. J. Biol. Chem. 282:37026-35

65. Han GS, Wu WI, Carman GM. 2006. The Saccharomyces cerevisiae Lipin homolog is a Mg2+dependent phosphatidate phosphatase enzyme. J. Biol. Chem. 281:9210-8

66. Harris TE, Finck BN. 2011. Dual function lipin proteins and glycerolipid metabolism. Trends Endocrinol. Metab. 22:226-33

67. Hayashi M, Toriyama K, Kondo M, Nishimura M. 1998. 2,4-dichlorophenoxybutyric acid-resistant mutants of Arabidopsis have defects in glyoxysomal fatty acid beta-oxidation. Plant Cell 10:18395 
68. He YH, Gan SS. 2002. A gene encoding an acyl hydrolase is involved in leaf senescence in Arabidopsis. Plant Cell 14:805-15

69. Henry SA, Kohlwein SD, Carman GM. 2012. Metabolism and regulation of glycerolipids in the yeast Saccharomyces cerevisiae. Genetics 190:317-49

70. Hernandez ML, Whitehead L, He ZS, Gazda V, Gilday A, et al. 2012. A cytosolic acyltransferase contributes to triacylglycerol synthesis in sucrose-rescued Arabidopsis seed oil catabolism mutants. Plant Physiol. 160:215-25

71. Horn PJ, James CN, Gidda SK, Kilaru A, Dyer JM, et al. 2013. Identification of a new class of lipid droplet-associated proteins in plants. Plant Physiol. 162:1926-36

72. James CN, Horn PJ, Case CR, Gidda SK, Zhang D, et al. 2010. Disruption of the Arabidopsis CGI-58 homologue produces Chanarin-Dorfman-like lipid droplet accumulation in plants. PNAS 107:17833-8

73. Jefferson T. 1800. Summary of Public Service. The Thomas Jefferson Papers Series 1. General Correspondence. 1651-1827 September:2

74. Jessen D, Roth C, Wiermer M, Fulda M. 2015. Two activities of long-chain acyl-coenzyme A synthetase are anvolved in lipid trafficking between the endoplasmic reticulum and the plastid in Arabidopsis. Plant Physiol. 167:351-U575

75. Kachroo A, Shanklin J, Whittle E, Lapchyk L, Hildebrand D, Kachroo P. 2007. The Arabidopsis stearoyl-acyl carrier protein-desaturase family and the contribution of leaf isoforms to oleic acid synthesis. Plant Mol. Biol. 63:257-71

76. Kalt-Torres W, Kerr PS, Usuda H, Huber SC. 1987. Diurnal changes in maize leaf photosynthesis : I. Carbon exchange rate, assimilate export rate, and enzyme activities. Plant Physiol. 83:283-8

77. Kammerer B, Fischer K, Hilpert B, Schubert S, Gutensohn M, et al. 1998. Molecular characterization of a carbon transporter in plastids from heterotrophic tissues: the glucose 6phosphate/phosphate antiporter. Plant Cell 10:105-17

78. Karim EK, Stephanie B, Emilia O, Anne-Marie G, Natalie F, Vincent A. 2005. Identification and characterization of a triacylglycerol lipase in Arabidopsis homologous to mammalian acid lipases. FEBS letters 579:6067-73

79. Katavic V, Reed DW, Taylor DC, Giblin EM, Barton DL, et al. 1995. Alteration of Seed Fatty-Acid composition by an ethyl methanesulfonate-induced mutation in Arabidopsis thaliana affecting diacylglycerol acyltransferase activity. Plant Physiol. 108:399-409

80. Ke J, Wen TN, Nikolau BJ, Wurtele ES. 2000. Coordinate regulation of the nuclear and plastidic genes coding for the subunits of the heteromeric acetyl-coenzyme A carboxylase. Plant Physiol. 122:1057-71

81. Kelly AA, Quettier AL, Shaw E, Eastmond PJ. 2011. Seed storage oil mobilization is important but not essential for germination or seedling establishment in Arabidopsis. Plant Physiol. 157:866-75

82. Kelly AA, Shaw E, Powers SJ, Kurup S, Eastmond PJ. 2012. Suppression of the SUGARDEPENDENT1 triacylglycerol lipase family during seed development enhances oil yield in oilseed rape (Brassica napus L.). Plant Biotechnol. J. 11: 355-61

83. Kelly AA, van Erp H, Quettier AL, Shaw E, Menard G, et al. 2013. The SUGAR-DEPENDENT1 lipase limits triacylglycerol accumulation in vegetative tissues of Arabidopsis. Plant Physiol. 162:1282-9

*Identifed SDP1 as the predominant TAG lipase in vegetative tissues.

84. Kim EY, Seo YS, Kim WT. 2011. AtDSEL, an Arabidopsis cytosolic DAD1-like acylhydrolase, is involved in negative regulation of storage oil mobilization during seedling establishment. J. Plant Physiol. 168:1705-9

85. Kim HU, Jung SJ, Lee KR, Kim EH, Lee SM, et al. 2013. Ectopic overexpression of castor bean LEAFY COTYLEDON2 (LEC2) in Arabidopsis triggers the expression of genes that encode 
regulators of seed maturation and oil body proteins in vegetative tissues. FEBS Open Bio. 4:2532

86. Kim HU, Lee KR, Jung SJ, Shin HA, Go YS, et al. 2015. Senescence-inducible LEC2 enhances triacylglycerol accumulation in leaves without negatively affecting plant growth. Plant Biotechnol. J. In press

87. Kim YY, Jung KW, Yoo KS, Jeung JU, Shin JS. 2011. A stress-responsive caleosin-like protein, AtCLO4, acts as a negative regulator of ABA responses in Arabidopsis. Plant Cell Physiol. 52:87484

88. Kjellberg JM, Trimborn M, Andersson M, Sandelius AS. 2000. Acyl-CoA dependent acylation of phospholipids in the chloroplast envelope. Biochim. Biophys. Acta 1485:100-10

89. Kohlwein SD. 2010. Triacylglycerol homeostasis: Insights from yeast. J. Biol. Chem. 285:15663-7

90. Kohlwein SD, Veenhuis M, van der Klei IJ. 2013. Lipid droplets and peroxisomes: Key players in cellular lipid homeostasis or A matter of fat-store 'em up or burn 'em down. Genetics 193:1-50

91. Kolling K, Thalmann M, Muller A, Jenny C, Zeeman SC. 2015. Carbon partitioning in Arabidopsis thaliana is a dynamic process controlled by the plants metabolic status and its circadian clock. Plant Cell Environ In press

92. Koo AJ, Fulda M, Browse J, Ohlrogge JB. 2005. Identification of a plastid acyl-acyl carrier protein synthetase in Arabidopsis and its role in the activation and elongation of exogenous fatty acids. Plant J. 44:620-32

93. Kunz HH, Scharnewski M, Feussner K, Feussner I, Flugge UI, et al. 2009. The ABC Transporter PXA1 and peroxisomal beta-oxidation are vital for metabolism in mature leaves of Arabidopsis during extended darkness. Plant Cell 21:2733-49

94. Lager I, Yilmaz JL, Zhou XR, Jasieniecka K, Kazachkov M, et al. 2013. Plant acyl-CoA: lysophosphatidylcholine acyltransferases (LPCATs) have different specificities in their forward and reverse reactions. J. Biol. Chem. 288:36902-14

95. Landolt R, Matile P. 1990. Glyoxisome-like microbodies in senescent spinach leaves. Plant Sci. 72:159-63

96. Lass A, Zimmermann R, Haemmerle G, Riederer M, Schoiswohl G, et al. 2006. Adipose triglyceridelipase-mediated lipolysis of cellular fat stores is activated by CGI-58 and defective in Chanarin-Dorfman Syndrome. Cell Metab. 3:309-19

97. Lersten NR, Czlapinski AR, Curtis JD, Freckmann R, Horner HT. 2006. Oil bodies in leaf mesophyll cells of angiosperms: Overview and a selected survey. Am. J. Bot. 93:1731-9

98. Li FL, Asami T, Wu XZ, Tsang EWT, Cutler AJ. 2007. A putative hydroxysteroid dehydrogenase involved in regulating plant growth and development. Plant Physiol. 145:87-97

99. Li H, Culligan K, Dixon RA, Chory J. 1995. CUE1: A mesophyll cell-specific positive regulator of light-controlled gene expression in Arabidopsis. Plant Cell 7:1599-610

100. Li N, Gugel IL, Giavalisco P, Zeisler V, Schreiber L, et al. 2015. FAX1, a novel membrane protein mediating plastid fatty acid export. Plos Biol. 13

101. Li R, Yu K, Hildebrand DF. 2010. DGAT1, DGAT2 and PDAT expression in seeds and other tissues of epoxy and hydroxy fatty acid accumulating plants. Lipids 45:145-57

102. Li Y, Beisson F, Pollard M, Ohlrogge J. 2006. Oil content of Arabidopsis seeds: the influence of seed anatomy, light and plant-to-plant variation. Phytochem. 67:904-15

103. Linka N, Theodoulou FL, Haslam RP, Linka M, Napier JA, et al. 2008. Peroxisomal ATP import is essential for seedling development in Arabidopsis thaliana. Plant Cell 20:3241-57

104. Lippold F, vom Dorp K, Abraham M, Holzl G, Wewer V, et al. 2012. Fatty acid phytyl ester synthesis in chloroplasts of Arabidopsis. Plant Cell 24:2001-14

105. Liu Q, Siloto RM, Lehner R, Stone SJ, Weselake RJ. 2012. Acyl-CoA:diacylglycerol acyltransferase: molecular biology, biochemistry and biotechnology. Prog. Lipid Res. 51:350-77 
106. Liu X, Sheng J, Curtiss R, 3rd. 2011. Fatty acid production in genetically modified cyanobacteria. PNAS 108:6899-904

107. Lock YY, Snyder CL, Zhu W, Siloto RM, Weselake RJ, Shah S. 2009. Antisense suppression of type 1 diacylglycerol acyltransferase adversely affects plant development in Brassica napus. Physiol. Plant. 137:61-71

108. Loewen CJ. 2012. Lipids as conductors in the orchestra of life. F1000 Biol. Rep. 4:4

109. Lonien J, Schwender J. 2009. Analysis of metabolic flux phenotypes for two Arabidopsis mutants with severe impairment in seed storage lipid synthesis. Plant Physiol. 151:1617-34

110. Lu CFL, de Noyer SB, Hobbs DH, Kang JL, Wen YC, et al. 2003. Expression pattern of diacylglycerol acyltransferase-1, an enzyme involved in triacylglycerol biosynthesis, in Arabidopsis thaliana. Plant Mol. Biol. 52:31-41

111. Lu SY, Song T, Kosma DK, Parsons EP, Rowland O, Jenks MA. 2009. Arabidopsis CER8 encodes LONG-CHAIN ACYL-COA SYNTHETASE 1 (LACS1) that has overlapping functions with LACS2 in plant wax and cutin synthesis. Plant J. 59:553-64

112. Ma W, Kong Q, Grix M, Mantyla JJ, Yang Y, et al. 2015. Deletion of a C-terminal intrinsically disordered region of WRINKLED1 affects its stability and enhances oil accumulation in Arabidopsis. Plant J. In press

113. Maeo K, Tokuda T, Ayame A, Mitsui N, Kawai T, et al. 2009. An AP2-type transcription factor, WRINKLED1, of Arabidopsis thaliana binds to the AW-box sequence conserved among proximal upstream regions of genes involved in fatty acid synthesis. Plant J. 60:476-87

114. Martin S, Parton RG. 2006. Lipid droplets: a unified view of a dynamic organelle. Nat. Rev. Mol. Cell Biol. 7:373-8

115. Masaki T, Mitsui N, Tsukagoshi H, Nishii T, Morikami A, Nakamura K. 2005. ACTIVATOR of Spomin::LUC1/WRINKLED1 of Arabidopsis thaliana transactivates sugar-inducible promoters. Plant Cell Physiol. 46:547-56

116. Mitra MS, Chen Z, Ren H, Harris TE, Chambers KT, et al. 2013. Mice with an adipocyte-specific lipin 1 separation-of-function allele reveal unexpected roles for phosphatidic acid in metabolic regulation. PNAS 110:642-7

117. Morandini P. 2013. Control limits for accumulation of plant metabolites: brute force is no substitute for understanding. Plant Biotechnol. J. 11:253-67

118. Murphy DJ. 2001. The biogenesis and functions of lipid bodies in animals, plants and microorganisms. Prog. Lipid Res. 40:325-438

119. Nakamura Y, Koizumi R, Shui G, Shimojima M, Wenk MR, et al. 2009. Arabidopsis lipins mediate eukaryotic pathway of lipid metabolism and cope critically with phosphate starvation. PNAS 106:20978-83

120. Napier JA. 2007. The production of unusual fatty acids in transgenic plants. Annu. Rev. Plant Biol. 58:295-319

121. Napier JA, Haslam RP, Beaudoin F, Cahoon EB. 2014. Understanding and manipulating plant lipid composition: Metabolic engineering leads the way. Curr. Opin. Plant Biol. 19:68-75

122. Niewiadomski P, Knappe S, Geimer S, Fischer K, Schulz B, et al. 2005. The Arabidopsis plastidic glucose 6-phosphate/phosphate translocator GPT1 is essential for pollen maturation and embryo sac development. Plant Cell 17:760-75

123. O'Grady J, Schwender J, Shachar-Hill Y, Morgan JA. 2012. Metabolic cartography: experimental quantification of metabolic fluxes from isotopic labelling studies. J. Exp. Bot. 63:2293-308

124. Ogas J, Kaufmann S, Henderson J, Somerville C. 1999. PICKLE is a CHD3 chromatin-remodeling factor that regulates the transition from embryonic to vegetative development in Arabidopsis. PNAS 96:13839-44 
125. Ohlrogge J, Allen D, Berguson B, DellaPenna D, Shachar-Hill Y, Stymne S. 2009. Driving on biomass. Science 324:1019-20

126. Ohlrogge J, Browse J. 1995. Lipid biosynthesis. Plant Cell 7:957-70

127. Ohlrogge J, Chapman KD. 2011. The seeds of green energy - expanding the contribution of plant oils as biofuels. The Biochemist 33:34-8

128. Ohlrogge JB, Jaworski JG. 1997. Regulation of fatty acid synthesis. Annu. Rev. Plant Physiol. Plant Mol. Biol. 48:109-36

129. Olofsson SO, Bostrom P, Andersson L, Rutberg M, Perman J, Boren J. 2009. Lipid droplets as dynamic organelles connecting storage and efflux of lipids. Biochim. Biophys. Acta 1791:448-58

130. Padham AK, Hopkins MT, Wang TW, McNamara LM, Lo M, et al. 2007. Characterization of a plastid triacylglycerol lipase from arabidopsis. Plant Physiol. 143:1372-84

131. Park S, Gidda SK, James CN, Horn PJ, Khuu N, et al. 2013. The alpha/beta hydrolase CGI-58 and peroxisomal transport protein PXA1 coregulate lipid homeostasis and signaling in Arabidopsis. Plant Cell 25:1726-39

132. Pascual F, Carman GM. 2013. Phosphatidate phosphatase, a key regulator of lipid homeostasis. Biochim. Biophys. Acta 1831:514-22

133. Petrie JR, Vanhercke T, Shrestha P, El Tahchy A, White A, et al. 2012. Recruiting a new substrate for triacylglycerol synthesis in plants: The monoacylglycerol acyltransferase pathway. Plos One 7

134. Prabhakar V, Lottgert T, Geimer S, Dormann P, Kruger S, et al. 2010. Phosphoenolpyruvate provision to plastids is essential for gametophyte and sporophyte development in Arabidopsis thaliana. Plant Cell 22:2594-617

135. Prabhakar V, Lottgert T, Gigolashvili T, Bell K, Flugge UI, Hausler RE. 2009. Molecular and functional characterization of the plastid-localized Phosphoenolpyruvate enolase (ENO1) from Arabidopsis thaliana. FEBS letters 583:983-91

136. Rambold AS, Cohen S, Lippincott-Schwartz J. 2015. Fatty acid trafficking in starved cells: regulation by lipid droplet lipolysis, autophagy, and mitochondrial fusion dynamics. Dev. Cell 32:678-92

137. Rani SH, Krishna TH, Saha S, Negi AS, Rajasekharan R. 2010. Defective in cuticular ridges (DCR) of Arabidopsis thaliana, a gene associated with surface cutin formation, encodes a soluble diacylglycerol acyltransferase. J. Biol. Chem. 285:38337-47

138. Reynolds KB, Taylor MC, Zhou XR, Vanhercke T, Wood CC, et al. 2015. Metabolic engineering of medium-chain fatty acid biosynthesis in Nicotiana benthamiana plant leaf lipids. Front. Plant Sci. 6

139. Roesler K, Shintani D, Savage L, Boddupalli S, Ohlrogge J. 1997. Targeting of the Arabidopsis homomeric acetyl-coenzyme A carboxylase to plastids of rapeseeds. Plant Physiol. 113:75-81

140. Rolland F, Sheen J. 2005. Sugar sensing and signalling networks in plants. Biochem. Soc. Trans. 33:269-71

141. Routaboul JM, Benning C, Bechtold N, Caboche M, Lepiniec L. 1999. The TAG1 locus of Arabidopsis encodes for a diacylglycerol acyltransferase. Plant Physiol. Biochem. 37:831-40

142. Ruiz-Lopez N, Haslam RP, Usher SL, Napier JA, Sayanova O. 2013. Reconstitution of EPA and DHA biosynthesis in arabidopsis: iterative metabolic engineering for the synthesis of $n-3$ LC-PUFAs in transgenic plants. Metab. Eng .17:30-41

143. Ruuska SA, Girke T, Benning C, Ohlrogge JB. 2002. Contrapuntal networks of gene expression during Arabidopsis seed filling. Plant Cell 14:1191-206

144. Sanjaya, Durrett TP, Weise SE, Benning C. 2011. Increasing the energy density of vegetative tissues by diverting carbon from starch to oil biosynthesis in transgenic Arabidopsis. Plant Biotechnol. J. 9:874-83 * Demonstrates increased TAG accumulation by inhibition of starch synthesis. 
145. Sanjaya, Miller R, Durrett TP, Kosma DK, Lydic TA, et al. 2013. Altered lipid composition and enhanced nutritional value of Arabidopsis leaves following introduction of an algal diacylglycerol acyltransferase 2. Plant Cell 25:677-93

146. Santos-Mendoza M, Dubreucq B, Baud S, Parcy F, Caboche M, Lepiniec L. 2008. Deciphering gene regulatory networks that control seed development and maturation in Arabidopsis. Plant J. 54:608-20

147. Santos-Rosa H, Leung J, Grimsey N, Peak-Chew S, Siniossoglou S. 2005. The yeast lipin Smp2 couples phospholipid biosynthesis to nuclear membrane growth. EMBO J. 24:1931-41

148. Santos Mendoza M, Dubreucq B, Miquel M, Caboche M, Lepiniec L. 2005. LEAFY COTYLEDON 2 activation is sufficient to trigger the accumulation of oil and seed specific mRNAs in Arabidopsis leaves. FEBS Lett. 579:4666-70

149. Sasaki Y, Kozaki A, Hatano M. 1997. Link between light and fatty acid synthesis: thioredoxinlinked reductive activation of plastidic acetyl-CoA carboxylase. PNAS 94:11096-101

150. Schnurr J, Shockey J, Browse J. 2004. The acyl-CoA synthetase encoded by LACS2 is essential for normal cuticle development in Arabidopsis. Plant Cell 16:629-42

151. Schwender J, Hay JO. 2012. Predictive modeling of biomass component tradeoffs in Brassica napus developing oilseeds based on in silico manipulation of storage metabolism. Plant Physiol. 160:1218-36

152. Schwender J, Hebbelmann I, Heinzel N, Hildebrandt TM, Rogers A, et al. 2015. Quantitative multilevel analysis of central metabolism in developing oilseeds of Brassica napus during in vitro culture. Plant Physiol. 168: 828-48 *Mathematical approach to understanding the basis of oil accumulation in seeds.

153. Scott RW, Winichayakul S, Roldan M, Cookson R, Willingham M, et al. 2010. Elevation of oil body integrity and emulsion stability by polyoleosins, multiple oleosin units joined in tandem head-totail fusions. Plant Biotechnol. J. 8:912-27

154. Shi SB, Chen Y, Siewers V, Nielsen J. 2014. Improving production of malonyl coenzyme A-derived metabolites by abolishing Snf1-dependent aegulation of Acc1. Mbio. 5

155. Shimada TL, Hara-Nishimura I. 2010. Oil-body-membrane proteins and their physiological functions in plants. Biol. Pharm. Bull. 33:360-3

156. Shimada TL, Shimada T, Takahashi H, Fukao Y, Hara-Nishimura I. 2008. A novel role for oleosins in freezing tolerance of oilseeds in Arabidopsis thaliana. Plant J. 55:798-809

157. Shimada TL, Takano Y, Shimada T, Fujiwara M, Fukao Y, et al. 2014. Leaf oil body functions as a subcellular factory for the production of a phytoalexin in Arabidopsis. Plant Physiol. 164:105-18

158. Shintani DK, Ohlrogge JB. 1995. Feedback inhibition of fatty-acid synthesis in tobacco suspension cells. Plant J. 7:577-87

159. Shockey JM, Fulda MS, Browse JA. 2002. Arabidopsis contains nine long-chain acyl-coenzyme A synthetase genes that participate in fatty acid and glycerolipid metabolism. Plant Physiol. 129:1710-22

160. Siloto RMP, Findlay K, Lopez-Villalobos A, Yeung EC, Nykiforuk CL, Moloney MM. 2006. The accumulation of oleosins determines the size of seed oilbodies in Arabidopsis. Plant Cell 18:1961-74

161. Singh R, Kaushik S, Wang Y, Xiang Y, Novak I, et al. 2009. Autophagy regulates lipid metabolism. Nature 458:1131-5

162. Slocombe SP, Cornah J, Pinfield-Wells H, Soady K, Zhang Q, et al. 2009. Oil accumulation in leaves directed by modification of fatty acid breakdown and lipid synthesis pathways. Plant Biotechnol. J. 7:694-703

163. Sorokin HP. 1955. Mitochondria and spherosomes in the living epidermal cell. Am. J. Bot. 42:225-31 
164. Stahl U, Carlsson AS, Lenman M, Dahlqvist A, Huang B, et al. 2004. Cloning and functional characterization of a phospholipid:diacylglycerol acyltransferase from Arabidopsis. Plant Physiol. 135:1324-35

165. Stitt M, Zeeman SC. 2012. Starch turnover: pathways, regulation and role in growth. Curr. Opin. Plant Biol. 15:282-92

166. Stone SL, Kwong LW, Yee KM, Pelletier J, Lepiniec L, et al. 2001. LEAFY COTYLEDON2 encodes a B3 domain transcription factor that induces embryo development. PNAS 98:11806-11

167. Thazar-Poulot N, Miquel M, Fobis-Loisy I, Gaude T. 2015. Peroxisome extensions deliver the Arabidopsis SDP1 lipase to oil bodies. PNAS 112:4158-63

168. Thelen JJ, Ohlrogge JB. 2002. Both antisense and sense expression of biotin carboxyl carrier protein isoform 2 inactivates the plastid acetyl-coenzyme A carboxylase in Arabidopsis thaliana. Plant J. 32:419-31

169. Theodoulou FL, Eastmond PJ. 2012. Seed storage oil catabolism: a story of give and take. Curr. Opin. Plant Biol. 15:322-8

170. Theodoulou FL, Holdsworth M, Baker A. 2006. Peroxisomal ABC transporters. FEBS Lett. 580:1139-55

171. Tjellstrom H, Strawsine M, Ohlrogge JB. 2015. Tracking synthesis and turnover of triacylglycerol in leaves. J. Exp. Bot. 66:1453-61

172. Tjellstrom H, Strawsine M, Silva J, Cahoon EB, Ohlrogge JB. 2013. Disruption of plastid acyl:acyl carrier protein synthetases increases medium chain fatty acid accumulation in seeds of transgenic Arabidopsis. FEBS Lett. 587:936-42

173. Tjellstrom H, Yang Z, Allen DK, Ohlrogge JB. 2012. Rapid kinetic labeling of Arabidopsis cell suspension cultures: implications for models of lipid export from plastids. Plant Physiol. 158:60111

174. To A, Joubes J, Barthole G, Lecureuil A, Scagnelli A, et al. 2012. WRINKLED transcription factors orchestrate tissue-specific regulation of fatty acid biosynthesis in Arabidopsis. Plant Cell 24:5007-23

175. Troncoso-Ponce MA, Cao X, Yang ZL, Ohlrogge JB. 2013. Lipid turnover during senescence. Plant Sci. 205:13-9

176. Troncoso-Ponce MA, Nikovics K, Marchive C, Lepiniec L, Baud S. 2015. New insights on the organization and regulation of the fatty acid biosynthetic network in the model higher plant Arabidopsis thaliana. Biochimie in press

177. Ugrankar R, Liu YL, Provaznik J, Schmitt S, Lehmann M. 2011. Lipin is a central regulator of adipose tissue development and function in Drosophila melanogaster. Mol. Cell. Biol. 31:164656

178. Valdearcos M, Esquinas E, Meana C, Pena L, Gil-de-Gomez L, et al. 2012. Lipin-2 reduces proinflammatory signaling induced by saturated fatty acids in macrophages. J. Biol. Chem. 287:10894-904

179. Van der Graaff E, Schwacke R, Schneider A, Desimone M, Flugge UI, Kunze R. 2006. Transcription analysis of arabidopsis membrane transporters and hormone pathways during developmental and induced leaf senescence. Plant Physiol. 141:776-92

180. Vanhercke T, El Tahchy A, Liu Q, Zhou XR, Shrestha P, et al. 2014. Metabolic engineering of biomass for high energy density: oilseed-like triacylglycerol yields from plant leaves. Plant Biotechnol. J. 12:231-9 *Three gene approach for optimazing oil accumulation in tobacco leaves.

181. Vanhercke T, El Tahchy A, Shrestha P, Zhou XR, Singh SP, Petrie JR. 2013. Synergistic effect of WRI1 and DGAT1 coexpression on triacylglycerol biosynthesis in plants. FEBS Lett. 587:364-9

182. Vanhercke T, Petrie JR, Singh S. 2014. Energy densification in vegetative biomass through metabolic engineering. Biocat.. Agr. Biotechnol. 3:75-80 
183. Voelker T. 1996. Plant acyl-ACP thioesterases: chain-length determining enzymes in plant fatty acid biosynthesis. Genet. Eng. 18:111-33

184. Wahlroos T, Soukka J, Denesyuk A, Wahlroos R, Korpela T, Kilby NJ. 2003. Oleosin expression and trafficking during oil body biogenesis in tobacco leaf cells. Genesis 35:125-32

185. Wang LP, Shen WY, Kazachkov M, Chen GQ, Chen QL, et al. 2012. Metabolic nteractions between the Lands cycle and the Kennedy pathway of glycerolipid synthesis in Arabidopsis developing seeds. Plant Cell 24:4652-69

186. Wang Z, Benning C. 2012. Chloroplast lipid synthesis and lipid trafficking through ER-plastid membrane contact sites. Biochem. Soc. Trans. 40:457-63

187. Weber APM, Linka N. 2011. Connecting the Plastid: Transporters of the Plastid Envelope and Their Role in Linking Plastidial with Cytosolic Metabolism. Annu. Rev. Plant Biol. 62:53-77

188. Wilfling F, Haas JT, Walther TC, Farese RV. 2014. Lipid droplet biogenesis. Curr. Opin. Cell Biol. 29:39-45

189. Winichayakul S, Scott RW, Roldan M, Hatier JH, Livingston S, et al. 2013. In vivo packaging of triacylglycerols enhances Arabidopsis leaf biomass and energy density. Plant Physiol. 162:626-39

190. Wu HY, Liu C, Li MC, Zhao MM, Gu D, Xu YN. 2013. Effects of monogalactoglycerolipid deficiency and diacylglycerol acyltransferase overexpression on oil accumulation in transgenic tobacco. Plant Mol. Biol. Rep. 31:1077-88

191. Xu C, Fan J, Froehlich JE, Awai K, Benning C. 2005. Mutation of the TGD1 chloroplast envelope protein affects phosphatidate metabolism in Arabidopsis. Plant Cell 17:3094-110

192. Yamaguchi T, Osumi T. 2009. Chanarin-Dorfman syndrome: Deficiency in CGI-58, a lipid dropletbound coactivator of lipase. Biochim. Biophys. Acta 1791:519-23

193. Yang ZL, Ohlrogge JB. 2009. Turnover of fatty acids during natural senescence of Arabidopsis, Brachypodium, and Switchgrass and in Arabidopsis beta-oxidation mutants. Plant Physiol. 150:1981-9

194. Yazdanbakhsh N, Sulpice R, Graf A, Stitt M, Fisahn J. 2011. Circadian control of root elongation and $C$ partitioning in Arabidopsis thaliana. Plant Cell Environ. 34:877-94

195. Zale J, Jung JH, Kim JY, Pathak B, Karan R, et al. 2015. Metabolic engineering of sugarcane to accumulate energy-dense triacylglycerols in vegetative biomass. Plant Biotechnol. J. In press *Demonstrates feasability of increasing vegetative TAG accumulation strategy in monocot crop plant.

196. Zhang M, Fan J, Taylor DC, Ohlrogge JB. 2009. DGAT1 and PDAT1 acyltransferases have overlapping functions in Arabidopsis triacylglycerol biosynthesis and are essential for normal pollen and seed development. Plant Cell 21:3885-901

197. Zhang P, Takeuchi K, Csaki LS, Reue K. 2012. Lipin-1 phosphatidic phosphatase activity modulates phosphatidate levels to promote peroxisome proliferator-activated receptor gamma (PPARgamma) gene expression during adipogenesis. J. Biol. Chem. 287:3485-94

198. Zhao LF, Katavic V, Li FL, Haughn GW, Kunst L. 2010. Insertional mutant analysis reveals that long-chain acyl-CoA synthetase 1 (LACS1), but not LACS8, functionally overlaps with LACS9 in Arabidopsis seed oil biosynthesis. Plant J. 64:1048-58

199. Zimmermann R, Strauss JG, Haemmerle G, Schoiswohl G, Birner-Gruenberger R, et al. 2004. Fat mobilization in adipose tissue is promoted by adipose triglyceride lipase. Science 306:1383-6

200. Zolman BK, Silva ID, Bartel B. 2001. The Arabidopsis pxa1 mutant is defective in an ATP-binding cassette transporter-like protein required for peroxisomal fatty acid beta-oxidation. Plant Physiol. 127:1266-78 


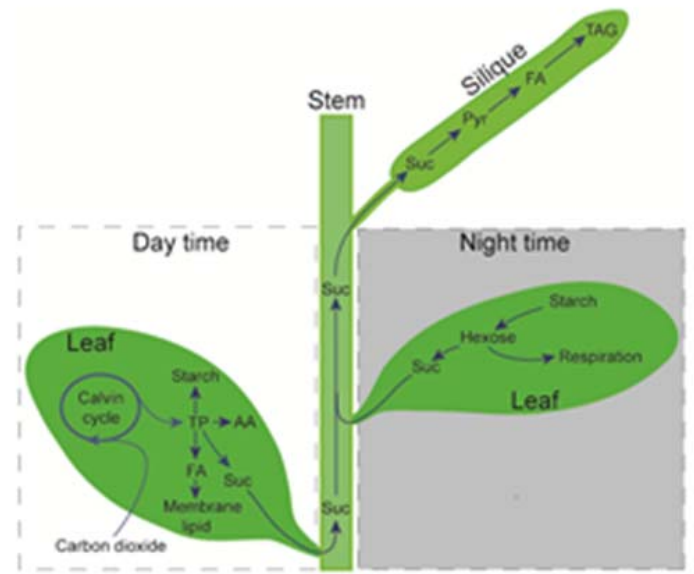

Figure 1 Overview of the source and sink relationship in oilseed plants. Additional abbreviation: AA, amino acids. 


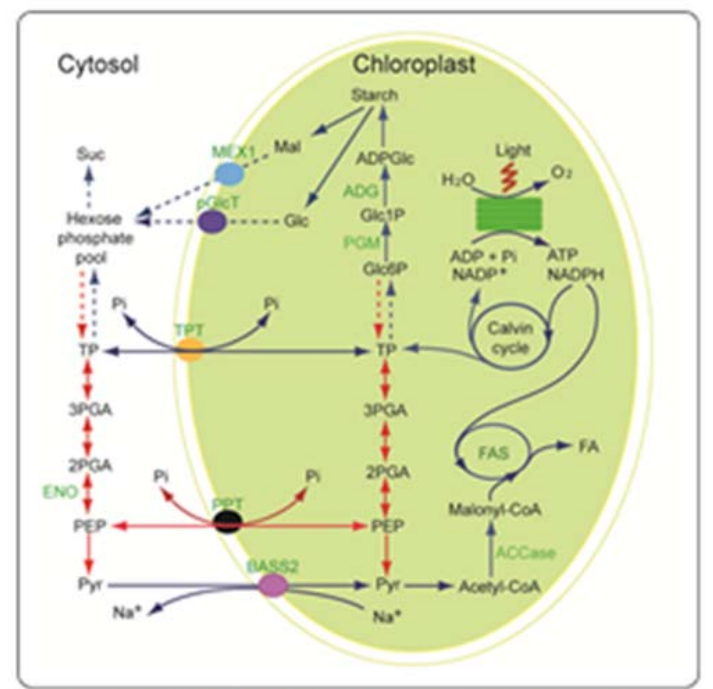

Figure 2. Overview of carbon flux in relation to FA and sucrose synthesis in a leaf mesophyll cell. Red arrows indicate steps in glycolysis. Photosynthetic light reactions generate ATP and DNAPH, which power both carbon fixation via the Calvin cycle and FA synthes is catalyzed by ACCase and a series of enzymatic reactions collectively referred to as fatty acid synthase (FAS). Dotted arrows indic ate multiple reactions. Green letters indicate proteins. Additional abbreviations: ADPGk, ADP_glucose; Glk1P, glucose-1phosphate; Glc 6 , glucose-6-phosphate; MEX1, maltose excess protein1; pGIcT, plastidial glucose transporter; 2PGA, 2-phosphoglycerate. 


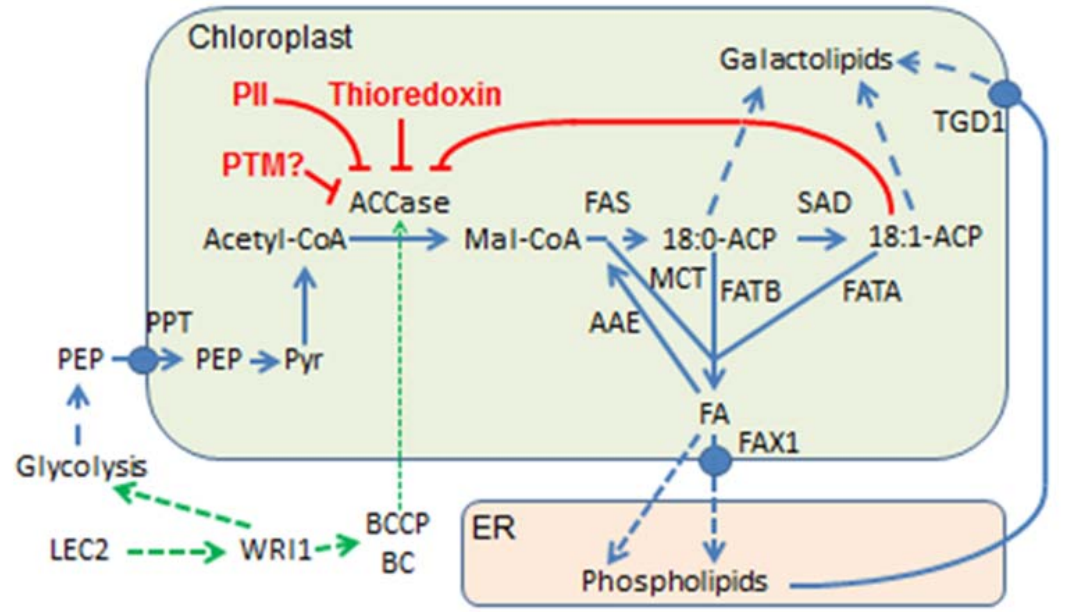

Figure 3. Scheme of factors that can potentially regulate the synthesis of FAs. 


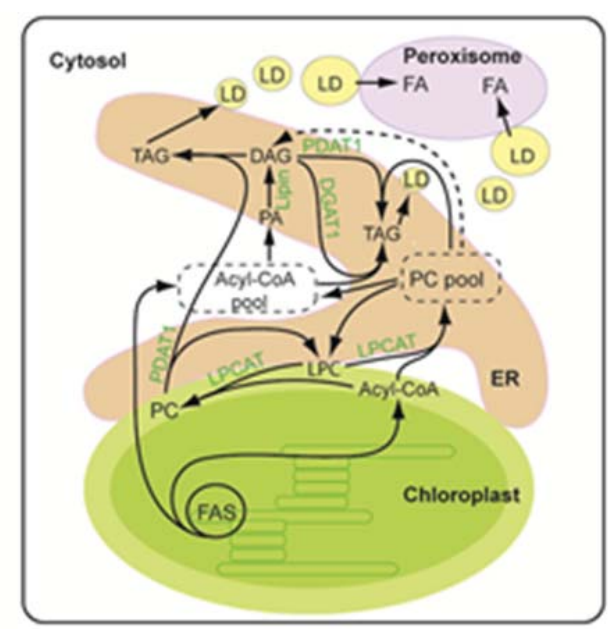

Figure 4 Overview of TAG metabolism pathways in a leaf mesophyll cell 